\title{
Effizienz von Smart Glasses: Ein Bewertungsmodell und Referenzwerte zur Bestimmung der Wirtschaftlichkeit
}

Sebastian Werning, Dennis Konusch und Ingmar Ickerott

Der betriebsinterne Einsatz von Smart Glasses ist bislang wenig erforscht, wodurch das Potenzial dieser Technologie noch nicht klar erkennbar ist. Die Digitalisierungsmaßnahmen in logistischen Prozessen und somit auch die Relevanz dieser Technologie soll im Zuge einer Wirtschaftlichkeitsbewertung analysiert werden. Mittels einer systematischen Literatur- und Anbieterrecherche werden hierfür Effizienz- und Investitionstreiber identifiziert. Eine Wirtschaftlichkeitsrechnung auf Basis von Prozessmodellen (WPM-Methode) wird um eine Zeiteinheitenanalyse erweitert. Diese soll die Vorteile des Einsatzes von Smart-Glasses-basierten Assistenzsystemen mithilfe von finanzwirtschaftlichen Kennzahlen darstellen können. Das hier verwendete Vorgehen untersucht den Kommissionierungsprozess als einen der ressourcenintensivsten Hauptprozesse der Logistik. Der Fokus liegt auf der Pick-by-Vision-Kommissioniermethode, da Wirtschaftlichkeitsuntersuchungen für diese Methode bisher nachrangig erforscht wurden. Durch die Ergänzung einer Investitionsrechnung können somit finanzwirtschaftliche Kennzahlen wie die Kapitalrentabilität ermittelt und mit alternativen Kommissioniermethoden verglichen werden. Das Bewertungsmodell ermöglicht die Wirtschaftlichkeitsbewertung einer neuartigen Technologie und regt in der Praxis zum Nachdenken über innovative Digitalisierungsmaßnahmen von logistischen Geschäftsprozessen an.

\section{Einleitung}

Die Integration von Smart Glasses trägt zur Digitalisierung von Arbeitsprozessen in der Logistik bei. Dabei werden häufig ressourcenintensive Prozesse betrachtet, da an dieser Stelle durch Digitalisierung eine Effizienzsteigerung herbeigeführt werden kann. Auf die Logistik bezogen handelt es sich bei dem Kommissionierprozess um einen der ressourcenintensivsten Prozesse, weshalb dieser für die nähere Betrachtung ausgewählt wurde. Die Smart-Glasses-basierte Lösung für einen Kommissionierungsprozess wird mit Pick-by-Vision betitelt. Diese Methode ist dafür bekannt, den Arbeitsprozess einer Kommissionierung zu optimieren. Oft- 
mals wird damit die Reduzierung des Zeitaufwands und eine geringere Fehlerquote in Verbindung gebracht (Günthner et al. 2009).

Insbesondere bei der freihändigen Ausführung informationsintensiver Tätigkeiten lassen sich solche Optimierungspotenziale realisieren. Aufgrund der dadurch steigenden Produktivität ergeben sich außerdem Kosteneinsparungspotenziale. Diese werden primär durch das Einblenden von Zusatzinformationen in das Sichtfeld des Kommissionierers ermöglicht. Die Informationsfreigabe wird durch ein an einem Brillengestell integriertes Display umgesetzt. Dadurch ermöglichen Smart Glasses mithilfe der Augmented Reality (AR = Erweiterte Realität) eine Prozessführung entlang des Kommissionierprozesses, welche die Reduzierung von Fehlerquoten ebenfalls fördert (Ludwig und Reimann 2005). Da ein solches System zunächst mit einem Investment verbunden ist, sich jedoch, wie soeben aufgezeigt, daraus auch monetäre Vorteile ergeben, ist es das Ziel dieses Beitrags, die Wirtschaftlichkeit von Smart-Glasses-basierten Assistenzsystemen in der Logistik zu untersuchen. Diese Einsatzpotenziale von Smart Glasses lassen sich ebenfalls als eine Rendite verstehen, die die Investition in Smart-Glasses-basierte Assistenzsysteme ermöglicht und im Verlauf dieses Beitrags als „Return“ zusammengefasst wird.

Das Ziel dieses Beitrags ist somit eine Wirtschaftlichkeitsbewertung von Smart-Glasses-basierten Assistenzsystemen in der Logistik. Wirtschaftlichkeit bedeutet im weiteren Sinne, mit den aufgewendeten Mitteln den größtmöglichen Ertrag zu erzielen oder für einen bestimmten Ertrag die geringstmöglichen Mittel einsetzen zu müssen. Dellmann und Pedell (1994) definieren diese Erkenntnis als umschriebenes Wirtschaftlichkeitsprinzip, das vielfältige Erscheinungsformen besitzt.

Um solchen Erscheinungsformen sowohl in der Theorie als auch in der Praxis zu begegnen, werden geeignete Methoden und Referenzwerte mithilfe einer systematischen Literatur- und (Smart-Glasses-) Anbieterrecherche ermittelt. Als Resultat liefert dieser Beitrag eine Hilfestellung für Unternehmen, um eine betriebsindividuelle Wirtschaftlichkeitsbewertung von Smart Glasses in der eigenen Logistik vornehmen zu können. Exemplarisch wird dabei die eingangs beschriebene Smart-Glasses-basierte Kommissioniermethode Pick-by-Vision betrachtet. Dadurch identifizierte Potenziale regen außerdem zur weiteren Forschung in der Wissenschaft von AR- und Smart-Glasses-basierten Technologien an.

Die Motivation hinter dem Vorhaben wird durch einen Megatrend begründet. Nicht nur als Trend bezeichnet, nimmt Digitalisierung von Geschäftsprozessen im Zuge des Zeitalters der Industrie 4.0 sowohl in Großunternehmen als auch in KMU an Wertschätzung zu (Ternès und Schieke 2018). Durch die Industrie 4.0 werden innovative Technologien in unternehmerische Arbeitsprozesse integriert, um durch eine industrielle Evolution entsprechende Wettbewerbsvorteile zu generieren. Unter innovativen Technologien fällt auch der Einsatz von AR. Digitalisierungsmaßnahmen in der Logistik lassen sich bereits an der mobilen Datenerfassung entlang des Kommissionierungsprozesses beobachten (ten Hompel et al. 2014). Dem Mangel an Fachliteratur und Langzeitbeobachtungen soll durch die- 
sen Beitrag in Form eines untersuchten Fallbeispiels entgegengewirkt werden. Weiterhin wird der Bedarf an Smart-Glasses-basierten Assistenzsystemen durch die Aussagen von Experten hinsichtlich des bevorstehenden Entwicklungsverlaufs in der Logistik positiv hervorgehoben (Böning und Kasselmann 2018). Erste Erkenntnisse aus der Fachliteratur zeigen, dass noch $50 \%$ der Lagersysteme in der Praxis auf „Mann-zur-Ware“-Lösungen basieren. Dies bedeutet, dass logistische Aufgaben zum großen Teil noch mit menschlicher Arbeitskraft ausgeführt werden. Deshalb ist es wichtig, geeignete und unterstützende Technologien für den Kommissionierprozess zu identifizieren. Hier können Smart-Glasses-basierte Systeme einen Mehrwert liefern (Almeida und Ferreira 2009).

Die Ermittlung von finanzwirtschaftlichen Kennzahlen in der Praxis zeigt, dass eine Wirtschaftlichkeitsbetrachtung und ein Bewertungsmodell für Smart-Glassesbasierte Systeme ihre Berechtigung haben. Als Ausgangslage werden repräsentative Kommissionierszenarien (KRS) herangezogen. Mithilfe dieser KRS kann ein Vergleich von Ist- und Soll-Prozessen durchgeführt werden und die Vorteile von Pick-by-Vision gegenüber alternativen Kommissioniermethoden in einer Prozessmodellierung dargestellt werden. Weiterhin wird durch Anwendung der TimeMeasurement-Methode oder auch MTM-Methode die Unterteilung der Prozesse in einzelne Teilprozesse möglich, wodurch diese vergleichbar werden (Almeida und Ferreira 2009). Die Ermittlung von Effizienztreibern auf Basis einer systematischen Literaturrecherche werden durch Referenzwerte aus der Praxis ergänzt, welche mithilfe einer Anbieterrecherche ermittelt worden sind.

Um eine umfassende Wirtschaftlichkeitsbewertung durchführen zu können, stellt der Beitrag folgende Forschungsfragen auf:

Forschungsfrage 1: Mit welcher Methode kann der Einsatz von Smart-Glassesbasierten Assistenzsystemen wirtschaftlich bewertet werden?

Ergänzend dazu die angelehnten Unterforschungsfragen:

- Welche Treiber der Wirtschaftlichkeit bestehen und wie werden diese ermittelt?

- Wie kann ein Unternehmer einschätzen, ob die Integration von Smart Glasses in seinem Lagersystem markttypisch ist?

Forschungsfrage 2: Mit welchen Kostentreibern ist bei Integration von SmartGlasses-basierten Assistenzsystemen in die betriebsinterne Struktur zu rechnen?

\section{Methodisches Vorgehen}

Um zu ermitteln, wie eine Wirtschaftlichkeitsbewertung von Smart Glasses innerhalb der Logistik umgesetzt werden kann, wurde zunächst eine systematische Literaturrecherche zwecks Identifikation von möglichen Effizienztreibern von Smart Glasses zur Wirtschaftlichkeitsbewertung durchgeführt. Diese wurde um eine Anbieterrecherche erweitert, da sich die Ergebnisse aus der Literaturrecherche oft- 
mals auf Labortests beziehen. Ergänzt wurde die Informationsgrundlage um einen Angebotsvergleich dreier unterschiedlicher Smart-Glasses-Anbieter. Dieser basiert auf drei unterschiedliche KRS und dient dem Zweck, Referenzwerte für die zugehörigen Investitionskosten zu identifizieren. Aus einem Pool an Methoden, wurde als Grundlage die WPM-Methode (Wirtschaftlichkeitsbewertung auf Basis von Prozessmodellen) nach Becker et al. (2012) ausgewählt, um eine betriebsindividuelle Anwendbarkeit zur Errechnung von finanzwirtschaftlichen Kennzahlen für Unternehmen zu ermöglichen (Becker et al. 2012). Der Vergleich zur Wirtschaftlichkeitsbewertung erfolgt zwischen der Pick-by-Vision-Methode und der Pickby-Scan-Methode, da es sich bei letzterer um eine der gängigsten Kommissioniermethoden handelt, bei der mittels eines MDE-Geräts (Mobiles Datenerfassungsgerät) kommissioniert wird (Reif 2009).

Zunächst wird ein Ist-Prozess modelliert, wodurch eine visuelle Darstellung der mit der Kommissionierung verbundenen Teilprozesse ermöglicht wird. Unter Anwendung der MTM-Methode kann anschließend ein Soll-Prozess simuliert werden (Galka und Ulbrich 2008). Die für diesen Beitrag ermittelten KRS umfassen unterschiedliche Lagerstrukturen. Unter Annahme einer Umsatzgenerierung durch Produktivitätssteigerung und einer monetären Bewertung von fehlerhaftem Greifen nach Artikeln im Kommissionierungsprozess (sog. Fehlpicks), können die erhobenen Effizienztreiber erstmals quantifiziert werden. Dadurch bieten Einsicht in das Einsparungspotenzial von Smart-Glasses-basierten Assistenzsystemen. Unter Erweiterung einer Investitionsrechnung um ein Kostenmodell, werden einmalige und laufende Kosten eines Angebotsvergleichs dreier Smart-Glasses-Anbieter berücksichtigt und zugehörige finanzwirtschaftliche Kennzahlen errechnet. Entsprechende Ergebnisse versuchen den Grad der Wirtschaftlichkeit der Smart Glasses in den jeweiligen Anwendungsfällen zu repräsentieren und können zum Vergleich in einem Benchmark herangezogen werden.

\subsection{Recherche}

\subsubsection{Systematische Literaturrecherche}

Zur Erhebung einer geeigneten Datengrundlage zur Durchführung einer Wirtschaftlichkeitsüberprüfung von Smart-Glasses-basierten Assistenzsystemen wurden eine Vielzahl von Datenbanken herangezogen. Hierzu dienten in erster Linie die Datenbanken: Sciencedirect, ISI Web of Knowledge, EBSCOhost, Springer, Emerald, Wiley online library, AIS electronic Library sowie Grauliteratur in Form von tagesaktuellen Printmedien und Internetrecherchen. Die Auswahl der Literatur erfolgte in Anlehnung an Webster \& Watsons Vorgehensweise zur Erstellung eines Literatur-Reviews, indem die Datenbanken nach einem bestimmten Term durchsucht werden (Webster und Watson 2016). Unter Anwendung des Terms (,Smart Glass“ OR „Smart Glasses“) AND („Kommissionierungsverfahren“ OR „Kommissionierung“ OR“ Logistik“ OR „Logistic“ OR „Wirtschaft“ OR „Economic“ OR „efficient)“ wurden insg. 538 Suchergebnisse ermittelt. Die identifi- 
zierte Literatur wurde in Hinblick auf ihre Relevanz bezogen auf die Wirtschaftlichkeit in der Logistik und den Einsatz von Smart Glasses systematisiert und ausgewählt. Einbezogen wurden Publikationen, die Methoden zur Bewertung der Wirtschaftlichkeit von Technologien beinhalten und Treiber der Wirtschaftlichkeit von Smart Glasses aufzeigen.

Diese Datengrundlage bezieht sich primär auf den Einsatz von Smart Glasses im Kommissionierungsprozess, da dieser oftmals als einer der ressourcenintensivsten Prozesse in der Logistik dargestellt wird (Tomkins et al. 2010). Tabelle 1 listet die Resultate der systematischen Literaturrecherche auf.

Tabelle 1. Ergebnisse der systematischen Literaturrecherche

\begin{tabular}{cccccccc}
\hline $\begin{array}{c}\text { Daten- } \\
\text { banken }\end{array}$ & Emerald & $\begin{array}{c}\text { Science } \\
\text { Direct }\end{array}$ & $\begin{array}{c}\text { Springer } \\
\text { Link }\end{array}$ & $\begin{array}{c}\text { Wiley } \\
\text { Online }\end{array}$ & $\begin{array}{c}\text { ISI Web } \\
\text { Knowledge }\end{array}$ & $\begin{array}{c}\text { AIS electr. } \\
\text { Library }\end{array}$ & $\begin{array}{c}\text { EBSCO- } \\
\text { host }\end{array}$ \\
\hline $\begin{array}{c}\text { Anzahl } \\
\text { Treffer }\end{array}$ & 24 & 276 & 68 & 76 & 22 & 22 & 50 \\
\hline
\end{tabular}

Gesamt: 538

Ausgeschlossen wurde Literatur, die keinen Fokus auf die Logistikbranche hat und bei der ein anderer inhaltlicher Schwerpunkt, z. B. Ergonomie, gesetzt ist. Es wurde nur Literatur verwendet, die frei zur Verfügung steht.

\subsubsection{Experteninterviews und Anbieterrecherche}

Im Rahmen der systematischen Literaturrecherche wurden bereits Effizienztreiber zur Bewertung der Wirtschaftlichkeit identifiziert. Dabei fällt auf, dass die meisten Untersuchungen auf Basis von Labortests stattgefunden haben und somit einen sehr geringen Anteil an Langzeitbeobachtungen aus der Praxis darstellen. Um die Wirtschaftlichkeit von Smart Glasses möglichst praxisnah bewerten zu können, wird die Recherche um eine Anbieterrecherche bzw. Experteninterviews erweitert. Hierzu werden die Anbieter von Smart Glasses befragt, um Effizienztreibern und Referenzwerte aus der Praxis zu ermitteln. Oftmals handelte es sich um übliche Verkaufs- und Beratungsgespräche, in denen Vor- und Nachteile von Smart Glasses geschildert werden konnten. In Anlehnung an (Mieg und Brunner 2001) wird in nachstehender Reihenfolge vorgegangen:

1. Schaffung einer Informationsgrundlage,

2. Erstellung eines Interview-Fragenkatalogs.

Zur Schaffung einer Informationsgrundlage in Punkt eins werden eine systematische Literaturrecherche und Ergebnisse aus themenbezogenen Publikationen der Universität und Hochschule Osnabrück herangezogen. Für den Fragenkatalog wird ein Aufbau aus drei Hauptfragen zur Strukturierung des Interviews gewählt.

- Frage 1: Welche Kommissionierverfahren finden derzeit am Markt in der Logistik-Branche am häufigsten Einsatz? Welche Branche bzw. Lagergröße sind am besten geeignet und warum? 
- Frage 2: Gibt es diesbezüglich Referenzwerte, bspw. in Form einer erhöhten Arbeitsgeschwindigkeit des Pick-by-Vision-Verfahrens gegenüber dem Pickby-Paper-Verfahren oder der Arbeitsgeschwindigkeit vom Pick-by-Vision-Verfahren gegenüber dem Pick-by-Scan-Verfahren?

- Frage 3: Wurden grundsätzlich Informationen bzgl. Praxistests von Smart Glasses in Logistikunternehmen erhoben? Wenn dem so ist, wie sind diese ausgefallen?

Nachdem der Fragenkatalog erstellt wurde, werden die zu befragenden Experten ausgewählt. Diese umfassen diverse Smart-Glasses-Anbieter. Diese wurden mittels eines Besuches einer Logistik-Messe (CeMAT 2018 in Hannover) und einer Websuche ermittelt und ermöglichen einen handelsüblichen Angebotsvergleich.

Während der Planung der Datenerhebung wurde ein „Vortest“ durchgeführt, indem die Anbieter im Vorfeld per E-Mail kontaktiert wurden. Diese erhielten bereits den beschriebenen Fragenkatalog. In den meisten Fällen wurden ausreichend Daten zu Verfügung gestellt, sodass kein persönliches Interview mehr folgen musste. In weiteren Fällen wurde nach Eingang der E-Mail beim Anbieter ein telefonisches Interview geführt, indem Antworten auf die obigen Fragen dokumentiert wurden.

\subsubsection{Angebotsvergleich}

Mithilfe eines Angebotsvergleichs dreier Anbieter, die sich auf Fachmessen als Anbieter für Smart-Glasses-basierte Logistiklösungen präsentiert haben, verfolgt das Forschungsvorhaben das Ziel, marktübliche Investitionskostensätze zu erfassen. Dies ermöglicht einen Vergleich von Kosteneinsparungspotenzialen in der Anschaffung von Smart Glasses. Auf dieser Grundlage soll eine praxisorientierte Wirtschaftlichkeitsbewertung ermöglicht werden. Die Angebote wurden jeweils für drei repräsentative KRS eines mittelständischen Logistikunternehmens angefordert. Die KRS zeichnen sich durch einen jeweils unterschiedlichen Digitalisierungsgrad sowie die unterschiedliche Anzahl an benötigten Datenbrillen aus. Dadurch erhalten Unternehmen einen breiten Einordnungsrahmen für die Anwendung der Wirtschaftlichkeitsbewertung. Der Angebotsvergleich ist in Tabelle 12 detailliert dargestellt.

\subsection{Ergebnisse der Recherche}

\subsubsection{Kommissioniermethoden}

Als ein Ergebnis der Literaturrecherche können vier Kommissioniermethoden als relevant für die Praxis eingestuft und somit in den Vordergrund der weiterführenden Analyse gestellt werden:

- Pick-by-Paper, 
- Pick-by-Scan (via MDE-Gerät),

- Pick-by-Voice und

- Pick-by-Light.

Bei einer klassischen Kommissionierung, wie der Pick-by-Paper-Kommissioniermethode, wird zur Informationsbereitstellung ein Beleg in Papierform gedruckt. Der Kommissionierer entnimmt Ware aus logistischen Einrichtungen in einer vorgegebenen Reihenfolge. Kommt es dabei zu Differenzen oder Fehlern, werden diese auf dem Beleg notiert, sodass im Nachfolgeprozess ordnungsgemäß eine Rechnung o. Ä. erstellt werden kann (Anrold et al. 2008).

Bei beleglosen Vorgehensweisen wie der Pick-by-Scan-Kommissioniermethode wird der Beleg durch ein Display oder Leuchttaster ersetzt. Dabei werden klassischerweise MDE-Geräte verwendet, die der Kommissionierer in Form von Handhelds mit sich trägt. Bei alternativen beleglosen Methoden wie der Pick-byVoice-Kommissioniermethode werden Kopfhörer verwendet, um eine Auftragsübermittlung per Sprache zu gewährleisten (De Vries et al. 2016).

Die Pick-by-Light-Kommissioniermethode baut auf am Lagerregal angebrachten Leuchtmittel auf, die an der entsprechenden Entnahmestelle aufleuchten und den Kommissionierer hierdurch anleiten (Gleißner und Femerling 2008). Die Pick-by-Paper-Lösung ist für KMU häufig die wirtschaftlichste Lösung während die Pick-by-Scan-Methode ein gängiges System darstellt, das erste Digitalisierungsmaßnahmen im Unternehmen vorantreibt (Reif 2009). In diesem Beitrag wird somit der Fokus auf die bekanntesten beleglosen- und belegorientierten Kommissioniermethoden Pick-by-Paper und Pick-by-Scan gelegt und als Referenz die Kommissioniermethode Pick-by-Vision herangezogen.

\subsubsection{Effizienztreiber}

Sowohl in Anwendungsfällen aus der Praxis (Case Studies) als auch in der Fachliteratur werden als Vorteile von Smart Glasses zumeist die erhöhte Arbeitsgeschwindigkeit (AG) und eine Fehlerquotenreduzierung (FQ) genannt. Diese beiden Einflussfaktoren stellen somit die Treiber der Effizienz innerhalb einer Wirtschaftlichkeitsbewertung dar. Aus den ermittelten Quellen wird in Tabelle 2 und Tabelle 3 jeweils ein Mittelwert gebildet.

Im Bereich der AG der Pick-by-Paper-Kommissioniermethode kann ein Ausreißer beobachtet werden. Dieser stellt eine negative Arbeitsgeschwindigkeit (-115 \%) bei der Quelle (Günthner 2010) dar. Die Unterschiedlichkeit der ermittelten Effizienztreiber ist vergleichsweise als hoch zu bewerten. Die Ergebnislage weist somit darauf hin, dass es in der Forschung an Methoden bedarf, die das Endergebnis konsolidieren können.

Neutrale Bewertungsmodelle sollen objektive Einschätzungen über die tatsächliche Effizienz von Smart Glasses in Geschäftsprozessen ergeben. Da sich die oben aufgeführten Referenzwerte der Effizienztreiber auf Fachliteratur beziehen, wurde die Literaturrecherche um eine Anbieterrecherche ergänzt. Dadurch soll 
vermieden werden, dass sich das Bewertungsmodell ausschließlich auf Referenzwerte bezieht, die auf Grundlage von Labortests ermittelt worden sind. Mithilfe der Anbieterrecherche werden diese Werte durch Referenzen ersetzt, die durch den Einsatz von Smart Glasses ebenfalls Produktivitätssteigerungen oder Fehlerquotenreduzierungen verzeichnen konnten. Die Referenzen stammen zu einem Großteil von Logistikdienstleistern und produzierenden Unternehmen. Die Werte sind im Detail im Anhang A aufgeführt.

Tabelle 2. Literaturrecherche - Effizienztreiber beim Pick-by-Vision

\begin{tabular}{lccc}
\hline Quelle & $\begin{array}{c}\text { Pick-by-Vision vs. } \\
\text { Pick-by-Paper }(A G)\end{array}$ & $\begin{array}{c}\text { Pick-by-Vision vs. } \\
\text { Pick-Scan }(A G)\end{array}$ & $\begin{array}{c}\text { Pick-by-Vision } \\
(F Q)\end{array}$ \\
\hline Loske und Kaspar (2018) & $19 \%$ & $9-12 \%$ & - \\
Günthner (2010) & $-115 \%$ & - & 0,125 \\
Guo (2014) & $37 \%$ & - & 0,0075 \\
Weaver (2010) & $30 \%$ & - & 0,1 \\
Reif und Günthner (2009) & $4 \%$ & - & 0,12 \\
Herter (2014) & $19 \%$ & - & 0,12 \\
$\begin{array}{l}\text { Reif et al. (2009) } \\
\text { Reif et al. (2009) (Veränderte }\end{array}$ & $4 \%$ & - & 0,12 \\
$\begin{array}{l}\text { Ausgangssituation) } \\
\text { Günthner et al. (2009) }\end{array}$ & $19 \%$ & - & 0,12 \\
(Labortests) & $4 \%$ & - & 0,82 \\
$\begin{array}{l}\text { Günthner et al. (2009) } \\
\text { (Praxis) }\end{array}$ & $4 \%$ & $10,50 \%$ & 0,12 \\
\hline Durchschnitt & $15,56 \%$ & & 0,1836 \\
\hline
\end{tabular}

In Bezug auf die FQ sind in der Fachliteratur Werte zu finden, die in einem Bewertungsmodell verwertbar sind. Die Anbieterrecherche hingegen erzielte diesbezüglich nur unzureichende Ergebnisse. Grund dafür ist eine durchschnittliche Fehlerquote innerhalb der Pick-by-Vision Methode, die in der Praxis durchschnittlich mit Null bewertet worden ist. Es ist fraglich, ob dieser Wert der Realität entsprechen kann oder zu Marketingzwecke adaptiert worden ist. Daher werden die „Fehlerquote $=$ Null“ Angaben nicht in die Wertung miteinbezogen. Der Mittelwert von verwertbaren Referenzwerte in Bezug auf die Effizienztreiber ist im Anhang A ermittelt worden.

Tabelle 3. Durchschnitt der Referenzwerte für Pick-by-Scan und Pick-by-Paper gegenüber Pick-by-Vision

\begin{tabular}{lcc}
\hline \multicolumn{1}{c}{ Kommissioniermethoden } & $A G$ & $F Q$ \\
\hline Pick-by-Vision vs. Pick-by-Scan & $19,98 \%$ & ca. $12 \%$ \\
Pick-by-Vision vs. Pick-by-Paper & $25,39 \%$ & ca. $45 \%$ \\
\hline
\end{tabular}

Die ermittelten Effizienztreiber werden im Verlauf dieses Beitrags für die Bestimmung eines Return-on-Investment (kurz: RoI = Kapitalrentabilität) herangezogen. 


\subsubsection{Investitionstreiber}

Im Rahmen der Wirtschaftlichkeitsbewertung wird der Return den anfallenden Kosten gegenübergestellt. Diese lassen sich als Investitionskosten für die Integrierung eines Pick-by-Vision-Systems interpretieren. Hierfür wurde ein Angebotsvergleich unterschiedlicher Smart-Glasses-Anbieter durchgeführt. Das Ziel ist es dabei nicht, auf Basis eines bestimmten Kriterienkataloges den vermeintlich besten Anbieter herauszusuchen. Viel mehr wird ein Eindruck dahingehend gewährt, wie sich die Anbieter und deren Lösungen für Smart Glasses überschneiden bzw. voneinander abgrenzen. Ziel dieser Methode ist es, mithilfe des in der kommerziellen Lösung inbegriffenen Wissenstands in Bezug auf die Pick-by-Vision-Technologien eine Kostenstruktur zu entwickeln, welche in Abb. 1 dargestellt ist.

\begin{tabular}{|c|c|}
\hline \multicolumn{2}{|c|}{ Gesamtkosten } \\
\hline Einmalige Kosten & \multicolumn{1}{|c}{ Laufende Kosten } \\
\hline Integrationskosten \\
\hline Hard- Softwarekosten \\
\hline \\
\end{tabular}

Abb. 1. Kostenstruktur von Smart-Glasses-basierten Assistenzsystemen

Die Gesamtkosten setzen sich aus den einmalig anfallenden und aus den laufenden Kosten zusammen. Die Integrationskosten umfassen dabei Server- und Schnittstellenanpassungen sowie Kosten für Softwareimplementierungsmaßnahmen. Die Hard- und Softwarekosten beziehen sich auf die Smart-Glasses-Endgeräte, während die Lizenzkosten für die längerfristige Nutzung anbieterabhängig kalkuliert werden. Darunter fällt unter anderem auch die Support-/Service-Abwicklung. Diese kann sowohl einmalig bei Installation der IT-Schnittstellen als auch in Form von laufenden Wartungsarbeiten anfallen. Sonstige Kosten umfassen diverse Aufwendungen, die bspw. mit den Wartungsarbeiten durch externes Personal entstehen. Hierzu können Reisekosten oder die Buchung von optionalen Softwaremodulen gehören, die i.d.R. nicht bei Erstinstallation des Pick-by-Vision-Systems berücksichtigt werden. Diese fallen teilweise einmalig, oftmals jedoch als laufende Kosten an.

\subsubsection{Methoden zur Wirtschaftlichkeitsbewertung}

Die Recherchephase hat einen Pool aus Methoden ergeben, welche sich für die Wirtschaftlichkeitsbewertung von innovativen Technologien eignen. In Tabelle 4 werden die Methoden aufgelistet, die einen hohen Praxisbezug aufweisen und im Rahmen dieses Beitrags angewendet oder um individuelle Anforderungen erweitert worden sind. 
Tabelle 4. Methodenpool - Wirtschaftlichkeitsbewertung

\begin{tabular}{lcc}
\hline \multicolumn{1}{c}{ Methoden } & Angewendet & $\begin{array}{c}\text { Erweitert und } \\
\text { angewendet }\end{array}$ \\
\hline $\begin{array}{l}\text { Capability Maturity Model Integration } \\
\text { (CMMI-Modell) (O'Regan 2014) }\end{array}$ & \\
$\begin{array}{l}\text { Wirtschaftlichkeitsbewertung auf Basis von Prozess- } \\
\text { modellen (WPM-Methode) (Becker et al. 2012) }\end{array}$ & $\mathrm{x}$ & \\
$\begin{array}{l}\text { Methods-Time Measurement } \\
\text { (MTM-Methode) (Almeida und Ferreira 2009) }\end{array}$ & $\mathrm{x}$ & \\
$\begin{array}{l}\text { Balanced Scorecard (Matt et al. 2015) } \\
\text { Vierstufiges Wirtschaftlichkeitsmodell (Zilker 2001) }\end{array}$ & & $\mathrm{x}$ \\
$\begin{array}{l}\text { Angebotsvergleich von Smart-Glasses-Anbietern } \\
\text { (Hochschule Osnabrück) } \\
\text { Gerätevergleich (Harnisch und Speerli 2015) }\end{array}$ & $\mathrm{x}$ & \\
\hline
\end{tabular}

Die Wirtschaftlichkeitsbewertung auf Basis von Prozessmodellen (WPM-Methode) wird als Hauptmethode im vorliegenden Beitrag fokussiert. Diese Methode stellt eine einfache Vorgehensweise für betriebsindividuelle Anwendungen dar. Die Anwendung wird mithilfe einer Prozessmodellierung durchgeführt, welche grundsätzlich auch von Menschen verstanden werden kann, die nicht betriebswirtschaftlich geschult sind (Scheer et al. 1997). Als Ergänzung zur WPM-Methode wird die Methods-Time Measurement (MTM)-Methode herangezogen, die eine Zerlegung eines Hauptprozesses in einzelne Teilprozesse ermöglicht (vgl. Tab. 4).

Im folgenden Abschnitt werden weitere Methoden vorgestellt, die alternativ zur WPM-Methode Anwendung finden können. Das CMMI-Modell, das ein bekanntes Reifegradmodell darstellt, wird primär bei Softwareimplementierungen eingesetzt. Die individuelle Anpassbarkeit macht das Modell jedoch in verschiedensten Anwendungsfällen anwendbar (Benmoussa et al. 2015).

Die Grundidee der Balanced Scorecard basiert auf der Erweiterung finanzorientierter Kennzahlensysteme durch einen Pool an nicht-finanziellen Erfolgsfaktoren bzw. Leistungstreibern, die miteinander verknüpft werden und zur Strategiefindung beitragen (Kaplan und Norton 2000).

Das vierstufige Wirtschaftlichkeitsmodell nach Picot und Reichwald wendet ebenfalls keine nummerische oder algorithmische Methode an, sondern skizziert ein Bewertungsmodell, das den Nutzen eines Vorhabens in vier Perspektiven betrachtet. Diese umfassen die isolierte, subsystembezogene, gesamtorganisatorische und gesellschaftliche Wirtschaftlichkeit und formuliert entsprechend ein qualitatives Ergebnis (Picot und Reichwald 1984).

Der Gerätevergleich dient primär der Betrachtung der Hardware von unterschiedlichen Endgeräten mit ähnlicher Funktionalität. In diesem Fall würde es sich um unterschiedliche Hersteller von Smart Glasses handeln. Die erwartete Performance durch Smart-Glasses-basierte Assistenzsysteme ist gerade durch den permanenten Einsatz und der Abhängigkeit vom angebrachten Bildschirm als äußerst hardwarespezifisch zu bewerten. Im Rahmen von Labortests wurden bereits mehrere Smart Glasses unter gleichen Umständen von selben Anwendern getestet und 
unterschiedliche Produktivität gemessen. Gründe für die Abweichungen waren u.a. eine mangelnde Gebrauchstauglichkeit, wodurch Zeitaufwand in Form von ständigem „Nachrücken“ der Datenbrille oder durch Systemausfälle entstanden ist (Harnisch und Speerli 2015). Somit wird die Auswahl einer geeigneten Smart Glasses als essenziell betrachtet, um eine erwartete Pickleistung zu erbringen.

\section{Auswahl geeigneter Methoden}

\subsection{Das Modell WPM-Methoden-Standard: Vorstellung}

Die WPM-Methode sieht vor, eine Wirtschaftlichkeitsbewertung auf Grundlage der Modellierung des neu einzuführenden Prozesses vorzunehmen. Anschließend können die mit dem Prozess verbundenen Ein- und Auszahlungen verglichen werden (Becker et al. 2012). Dabei bezieht sich der Vorgang auf den Kommissionierungsprozess. Die Vorgehensweise wird nach Becker (2012) wie folgt aufgebaut:

1. Erfassung der Auszahlungen von Funktionen

2. Erfassung der Einzahlungen von Funktionen

3. Aggregation der Zahlungsfolge einer Prozessalternative

4. Kalkulation der finanzwirtschaftlichen Konsequenzen einer Prozessalternative

5. Berechnung von Wirtschaftlichkeitskennzahlen für den Vergleich von Prozessalternativen

Die Ergebnisse, basierend auf dem Vergleich eines Ist-Prozesses mit einer Alternative, werden zusammengefasst und ein Handlungsansatz abgeleitet. Dieser beinhaltet u.a. die Aufstellung von finanzwirtschaftlichen Kennzahlen für das Unternehmen. Abb. 2 visualisiert die klassische WPM-Methode nach Becker (2012).

\subsection{Das Modell WPM-Methode-Erweitert: Anwendung}

Die erweiterte WPM-Methode dient dem Zweck, einen Soll-Prozess zu simulieren. Unter der Verwendung von variablen Parameterwerten, kann ein Pick-by-Vision-Prozess simuliert und mit einem betriebsinternen Ist-Prozess verglichen werden. In Anlehnung an Becker (2012) wird die Vorgehensweise der WPM-Methode wie folgt modifiziert:

1. Definition und Beschreibung des zu beeinflussenden Prozesses

2. Prozessmodellierung des zu beeinflussenden Prozesses

3. Definition des Soll-Prozesses

4. Darstellung des Soll-Prozesses:

4.1. Erfassung der mit dem Prozess verbundenen Einzahlungen (Return)

4.2. Erfassung der mit dem Prozess verbundenen Auszahlungen (Kosten)

5. Ermittlung finanzwirtschaftlicher Kennzahlen

6. Vergleich der Ergebnisse und Handlungsansatz formulieren 


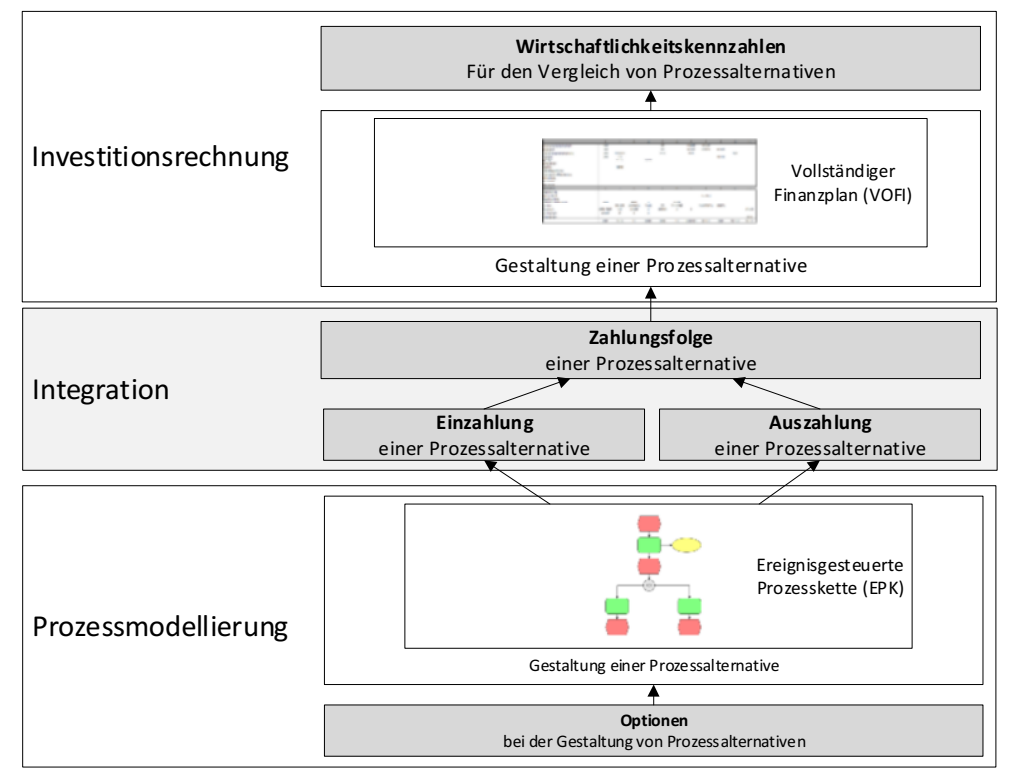

Abb. 2. Klassische WPM-Methode nach Becker (2012)

1. Definition und Beschreibung des zu beeinflussenden Prozesses: Der zu beeinflussende Prozess umfasst die Kommissionierung, welche das kundengerechte Zusammenstellen von Gütern aus einem Sortiment abwickelt. Dabei bezieht sich dieser Beitrag auf das KRS 1. Dieses basiert auf einem belegorientierten Kommissionierverfahren, das das MDE-Gerät nur teilweise integriert um einen schnelleren Datenaustausch zu gewährleisten. Mithilfe eines Papierauftrages entnimmt ein Kommissionierer Artikel aus einem Lagersystem und scannt die Ware bzw. das Lagerfach mit einem MDE-Gerät (Hompel et al. 2011). Beim Scannen von entsprechenden Artikeln kommuniziert das MDE-Gerät oftmals über WLAN (Wireless Local Area Network) mit einem Server oder Host. In der Regel wird das Gerät mit manuellen Tasten vom Kommissionierer bedient (Hompel et al. 2011). Durch diese Methode wird die belegorientierte Kommissionierungsmethode ergänzt und die Digitalisierung vorangetrieben (Richter 2018). Der vorliegende Fall beschreibt also eine Kombination aus Pick-by-Paper und Pick-by-Scan.

2. Prozessmodellierung des zu beeinflussenden Prozesses: Die Prozessmodellierung des zu beeinflussenden Prozesses orientiert sich an der klassischen Darstellung der Kommissionierung nach Reif (2009). Dabei werden die Hauptprozesse in Teilprozesse zerlegt und visuell dargestellt. Die in Abbildung 3 mit einem Kreis gekennzeichneten Teilprozesse stellen die Teilprozesse mit einem hohen Informationsaustausch dar. Diese beinhalten somit das Potenzial für Integration von Digitalisierungsmaßnahmen (Reif 2009). 


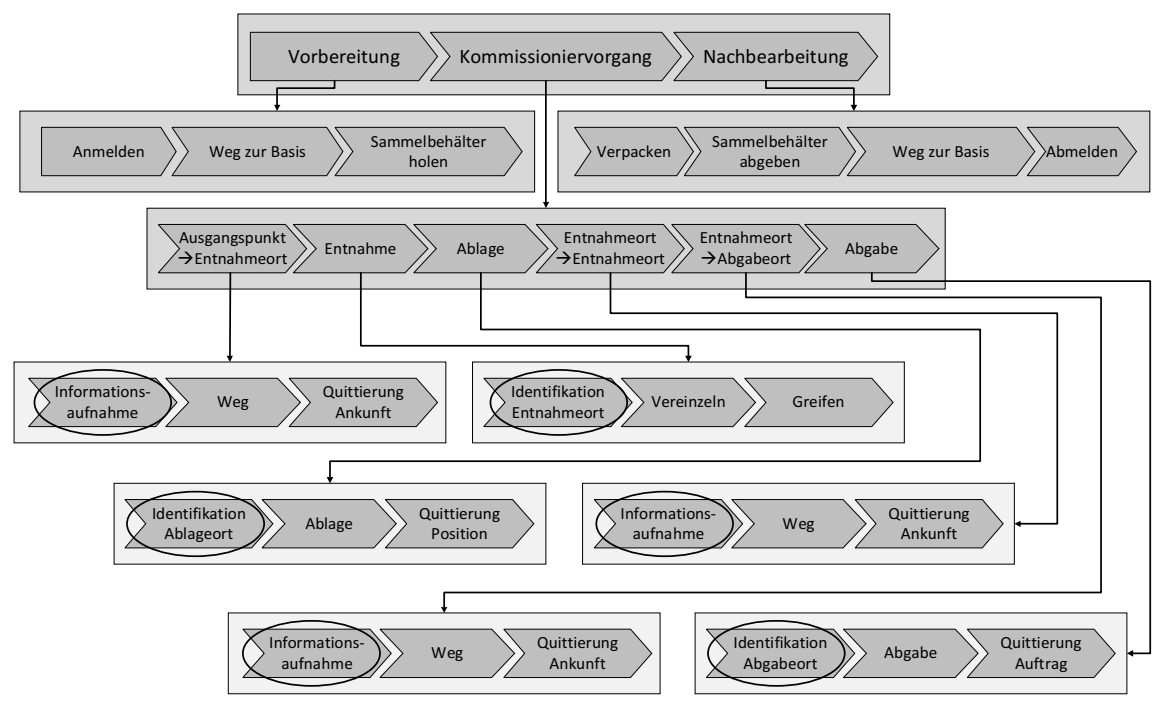

Abb. 3. Prozessmodellierung - Kommissionierung nach Reif (2009)

3. Definition des Soll-Prozesses: Der Soll-Prozess umfasst in diesem Fall die Pick-by-Vision-Kommissioniermethode. Diese Technologie versorgt den Träger einer Smart Glasses mit Informationen auf einem Display, sodass die reale Umwelt um virtuelle Inhalte „,erweitert“ wird. Die Smart Glasses integrieren den Einsatz von AR im Kommissionierungsprozess, indem eine situationsabhängige Informationsbereitstellung in visueller Form zur Leistungs- und Qualitätssteigerung genutzt wird. Somit ist eine stetige technische Unterstützung während des Kommissionierprozesses gegeben (Günthner et al. 2009).

4. Darstellung des Soll-Prozesses (Return/Kosten): Das Vorgehen zur Darstellung eines Soll-Prozesses wird in Arbeitspakete eingeteilt (vgl. Tabelle 5).

Tabelle 5. Ermittlung der mit dem Soll-Prozess verbundenen Ein- und Auszahlungen

\begin{tabular}{|c|c|c|}
\hline & Methode & Arbeitspakete $(A P)$ \\
\hline \multirow[t]{7}{*}{ Einzahlungen } & 1.0 & Darstellung des Ist-Prozesses in Teilprozesse \\
\hline & MTM-Methode & Prozessmodellierung Ist-Prozess \\
\hline & & $\begin{array}{l}\text { + Ableitung des Soll-Prozesses (Prozessmodellie- } \\
\text { rung) }\end{array}$ \\
\hline & & Bewertung der Teilprozesse mit Zeiteinheiten \\
\hline & & $\begin{array}{l}\text { Monetäre Bewertung von eingesparten Zeiteinhei- } \\
\text { ten (time-saving) }\end{array}$ \\
\hline & 2.0 & Ermittlung von Effizienztreibern \\
\hline & $\begin{array}{l}\text { Literaturrecherche } \\
+ \text { Anbieterrecherche }\end{array}$ & \\
\hline \multirow[t]{2}{*}{ Auszahlungen } & 3.0 & Repräsentative KRS \\
\hline & $\begin{array}{l}\text { Datengrundlage aus } \\
\text { dem Projekt GLASS- } \\
\text { HOUSE }\end{array}$ & Angebotsvergleich \\
\hline
\end{tabular}


Methode 1.0: MTM-Methode: Um Teilprozesse des Ist-Prozesses bestimmen und zeitliche Aufwandsminderungen monetär bewerten zu können, bietet sich die Anwendung der MTM-Methode an. Diese zeichnet sich durch einen bereits bekannten Lösungsalgorithmus aus und ermöglicht eine methodische Beschreibung eines Soll-Zustandes (Galka und Ulbrich 2008). Da die WPM-Methode auf eine Prozessmodellierung abzielt, kann in Kombination mit der MTM-Methode eine detaillierte Prozessmodellierung abgeleitet werden. Hierbei werden unter Teilprozesse auch einzelne Bewegungsabläufe und Grundbewegungen verstanden, wie bspw. hinlangen, greifen und heben. Da die MTM-Methode als eine geeignete Zeitermittlungsmethode genutzt werden kann, werden alle Teilprozesse unter Berücksichtigung der Auftrags- und Lagerstruktur mit Zeiteinheiten bewertet.

In der nachfolgenden Darstellung wird der Kommissionierungsprozess aus dem KRS 1 eines Logistik-Unternehmens betrachtet. Der Prozess wurde im Rahmen einer MTM-Methode in einzelne Teilprozesse zerlegt. Der beanspruchte Zeitaufwand dieser Teilprozesse wurde ebenfalls erfasst. In Anlehnung an eine Darstellung des Pick-by-Vision Grundprozesses nach Günthner (2009) und des KRS 1 können die Prozesse in folgender Abb. 4 verglichen werden.

Die Zeitbemessung der einzelnen Teilprozesse kann mithilfe einer MTM-Analyse ermittelt werden. In dem gewählten Beispiel orientiert sich die Analyse an den Prozess in einem Logistikunternehmen in der Fashionbranche. Die Zeitbemessung der einzelnen Teilprozesse bezieht sich auf eine durchschnittliche Anzahl an Auftragspositionen, die in der Prozesserhebung ermittelt wurden (vgl. Tabelle 6).

Tabelle 6. Ergebnis - MTM-Analyse, Pick-by-Vision vs. Pick-by-Paper/Scan, KRS 1

\begin{tabular}{cc}
\hline $\begin{array}{c}\text { Benötigter Zeitaufwand } \\
\text { pro Auftragseinheit: }\end{array}$ & $\begin{array}{c}\text { Benötigter Zeitaufwand } \\
\text { pro Auftragseinheit: } \\
\text { Pick-by-Vision }\end{array}$ \\
\hline Pick-by-Scan/Paper \\
\hline 128,11 Sekunden $=0,03$ Stunden & 197 Sekunden $=0,05$ Stunden \\
\hline
\end{tabular}

Ausgangslage: Aufgrund des im KRS 1 ermittelten Zeitaufwands pro durchschnittlicher Auftragseinheit, kann nun eine monetäre Bewertung vorgenommen werden. Hierfür wurde im Rahmen der KRS ermittelt, dass ein Kommissionierer für den Arbeitgeber durchschnittliche Kosten von $23 €$ pro Stunde verursacht. Weiterhin folgt die Annahme, dass ein Kommissionierer ca. vier Aufträge pro Stunde mit jeweils vier Positionen pro Auftrag abwickeln kann. Dabei gilt ein regulärer Arbeitstag mit acht Stunden Arbeitszeit. Im oben analysierten KRS sind zwei Kommissionierer beschäftigt, sodass entsprechend zwei Datenbrillen benötigt werden. Das Einsparungspotenzial ist in Tabelle 7 dargestellt.

Die Herleitung des Returns erfolgt auf Basis des Wirtschaftlichkeitsprinzips. Dieses umfasst entweder das Maximalprinzip, wodurch ein maximales Ergebnis bei gegebenem Input erreicht wird (z. B. mehr Picks bei gleichbleibender Arbeitszeit) oder das Minimalprinzip, wodurch ein gegebenes Ziel mit minimalem Input erreicht wird (z.B. bestimmter Auftragsdurchlauf bei minimalem zeitlichem Arbeitsaufwand) (Eichhorn und Merk 2016). 

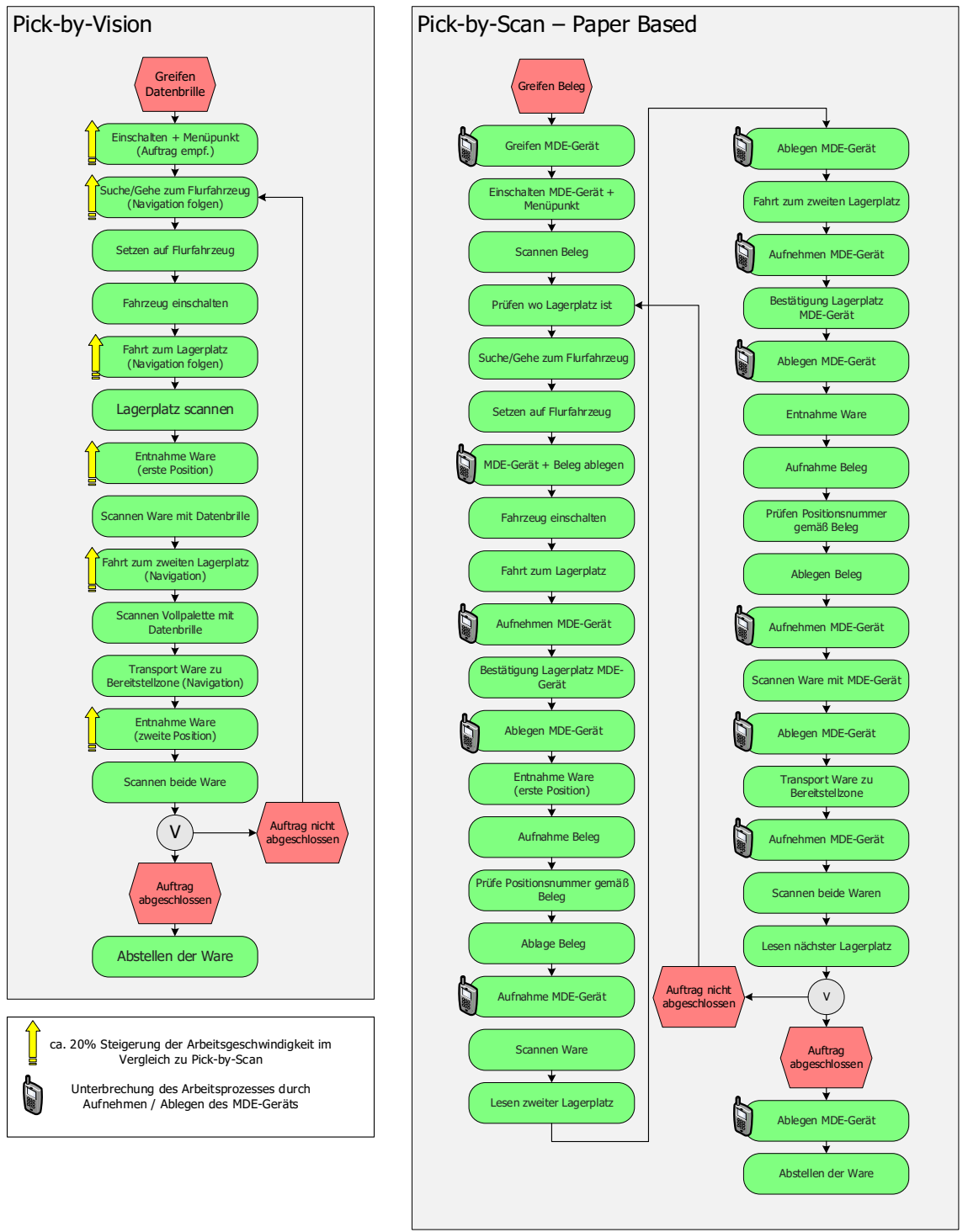

Abb. 4. Pick-by-Vision vs. Pick-by-Paper/Scan, KRS 1

Weiterhin wurden durchschnittliche Fehlerquoten und die damit verbundenen Kosten pro Fehlpick ermittelt, um eine monetäre Bewertung zu ermöglichen. In Tabelle 8 werden durchschnittliche Fehlerkosten aus der als relevant identifizierten Fachliteratur aufgelistet. 
Tabelle 7. Einsparungspotenzial - MTM-Methode; KRS 1

\begin{tabular}{|c|c|}
\hline Einsparungspotenzial & Ausgangslage (Annahme) \\
\hline gemessen in & - 4 Positionen / Auftrag - 4 Aufträge / Stunde \\
\hline Sek. / Stunden & $\begin{array}{l}\text { - } 8 \text { Stunden / Arbeitstag - } 2 \text { Kommissionierer mit jeweils } 23 \\
€ / \text { Stunde }\end{array}$ \\
\hline \multicolumn{2}{|l|}{ Rechnung } \\
\hline 197,00 Sek. $=0,05$ Stunden & $\begin{array}{l}4 \text { Aufträge x } 8 \text { Stunden }=32 \text { Aufträge } / \text { Tag; } \\
32 \text { Auftragspositionen x } 4 \text { Aufträge = } 128 \text { Auftragspositionen } \\
\text { / Tag; }\end{array}$ \\
\hline 128,11 Sek. = & 128 Auftragspositionen pro Tag x 0,02 Stunden (Differenz \\
\hline 0,03 Stunden & $0,05-0.03)=2,56$ Stunde Zeiteinsparung / Tag; \\
\hline (siehe Abb. 4/ & 2,56 Stunde Zeiteinsparung / Tag x \\
\hline Tabelle 6) & $\begin{array}{l}23 € / \text { Kommissioniererstundenlohn } \\
=58,88 € \text { Kosteneinsparung pro Kommissionierer mit } \\
\text { Datenbrille }\end{array}$ \\
\hline$=68,89 / 0,02$ & $58,88 €$ Einsparung am Tag / Datenbrille \\
\hline \multicolumn{2}{|c|}{$\begin{array}{l}58,88 € \times 2 \text { Kommissionierer mit Datenbrillen } \\
=117,76 € \text { Kosteneinsparung für zwei Kommissionierer mit Datenbrillen pro Tag } \\
117,76 € \text { Einsparung pro Tag in KRS } 1 \text { (zwei Datenbrillen) }\end{array}$} \\
\hline \multicolumn{2}{|c|}{$\begin{array}{l}\text { Ergebnis: } \text { Einfache Übersicht } \\
\text { Ist-Prozess (Pick-by-Scan): } 197 \text { Sekunden pro Auftragseinheit }=100 \% \\
\text { Soll-Prozess (Pick-by-Vision): } 128,11 \text { Sekunden pro Auftragseinheit = ca. } 65 \% \\
\text { Einsparungspotenzial = ca. } 35 \%\end{array}$} \\
\hline
\end{tabular}

Tabelle 8. Durchschnittliche Fehlerkosten in der Kommissionierung

\begin{tabular}{|c|c|c|}
\hline Quelle & Sachverhalt & Fehlerkosten \\
\hline Furmans und Linsel (2011) & Bei sofortiger Behebung eines Fehlers & $25 € /$ Fehler \\
\hline Stoll (2015) & Fehler wird erst beim Kunden entdeckt & $\begin{array}{c}210 € / \text { Fehler } \\
\text { (ca. } 10 \times 21 € / \\
\text { Fehler) }\end{array}$ \\
\hline \multirow{3}{*}{$\begin{array}{l}\text { xpert.digital (2016) } \\
\text { Crostack et al. (2008) }\end{array}$} & Jeder durchschnittliche Fehlgriff & $17 € /$ Fehler \\
\hline & $\begin{array}{l}\text { Gesamtkosten bei Fehlerquote entspricht } \\
32 \text { Fehler auf } 1000 \text { Positionen }\end{array}$ & $23,13 € /$ Fehler \\
\hline & Mittelwert & $21,53 € /$ Fehler \\
\hline
\end{tabular}

Nach Hausladen (2016) sind in einem belegorientieren Kommissionierverfahren bis zu 300 Picks pro Stunde möglich bzw. max. 2.400 Picks pro Tag (300 Picks x 8 Stunden).

Die in Tabelle 8 berücksichtigten Referenzwerte haben ebenfalls ergeben, dass auf Basis von Labor- und Praxistests eine Fehlerquotenreduzierung erzielt werden kann. Diese umfasst ca. $45 \%$ bei Pick-by-Vision- gegenüber eines belegorientierten Kommissionierverfahrens. Gegenüber eines Pick-by-Scan-Kommissionierverfahrens kann die Fehlerquotenreduzierung bis zu $12 \%$ betragen. Die Werte sind Tabelle 3 entnommen. Die Datengrundlage der entsprechenden Literaturrecherche kann im Anhang B nachvollzogen werden. Dadurch kann folgendes Kosteneinsparungspotenzial errechnet werden (vgl. Tabelle 9). 
Tabelle 9. Kosteneinsparung durch Fehlerquotenreduzierung

\begin{tabular}{|c|c|c|c|}
\hline & Pick-by-Vision & Pick-by-Paper & Pick-by-Scan \\
\hline $\begin{array}{l}\text { Fehlerquote } \\
\text { (Literaturrecherche) }\end{array}$ & $\begin{array}{l}0,1836 \% \\
\text { siehe Tabelle } 2\end{array}$ & $\begin{array}{l}0,1836 \% \times 45 \% \\
=0,27 \%\end{array}$ & $\begin{array}{l}0,1836 \% \times 12 \% \\
=0,21 \%\end{array}$ \\
\hline Kosten pro Fehler & $21,53 €$ & $21,53 €$ & 21,53 \\
\hline Fehlpicks/h bei 2.400 Picks/Tag & 5 & 7 & 6 \\
\hline $\begin{array}{l}\text { Fehlerkosten am Tag } \\
\text { (8 Stunden) }\end{array}$ & $107,65 €$ & $150,71 €$ & $129,18 €$ \\
\hline $\begin{array}{l}\text { Fehlerkosten im Jahr (300 Tage) } \\
\text { Kosteneinsparung }\end{array}$ & $32.295 €$ & $\begin{array}{l}45.213 € \\
+12.918 € / \mathrm{Jahr} \\
(+29 \%)\end{array}$ & $\begin{array}{l}38.753 € \\
+6.458 € / \mathrm{Jahr} \\
(+16 \%)\end{array}$ \\
\hline
\end{tabular}

Die Ermittlung finanzwirtschaftlicher Kennzahlen orientiert sich an etablierten Messgrößeren der Betriebswirtschaft. Am Beispiel des Return on Investment setzen sich zugehörige Kalkulationen zusammen aus den Investitionskosten und den Einsparungen bzw. dem „Return“. Letzterer kann sich aus der erhöhten AG und der reduzierten FQ ergeben. Außerdem wird die durchschnittliche Lebensdauer einer Smart Glasses berücksichtigt. Da es in der Forschung und Praxis an Langzeitbeobachtungen mangelt, wird eine durchschnittliche Lebensdauer von fünf Jahren angesetzt. Dieser Richtwert orientiert sich an der durchschnittlichen Lebensdauer von MDE-Geräten (Sommerhäuser 2017). Der RoI und die damit verbundene Amortisationsdauer lassen sich am Beispiel von Angebot 1 für KRS 1 wie folgt berechnen (Capone 2007):

$$
\begin{gathered}
R O I=\frac{\text { Gewinn }}{\text { eingesetztes Kapital }} \\
R O I=\frac{35.334 €}{39.670 €} \\
R O I=0,89 \\
\text { Amortisationsdauer }=\frac{\text { Kapitaleinsatz }}{\emptyset \text { Return pro Periode }} \\
\text { Amortisationsdauer }=\frac{39.670 €}{35.334 €} \\
\text { Amortisationsdauer }=1,12 \text { Jahre }
\end{gathered}
$$

Der ausgewiesene Gewinn (35.334 €) bzw. der erzielte Return pro Jahr berechnet sich aus der Einsparung pro Tag $(58,89 €)$, siehe Tabelle 8, multipliziert mit der Anzahl an Arbeitstragen (300 Tage), die für das KRS 1 angenommen wurden, und multipliziert mit der Anzahl an Datenbrillen (2 Stück), die für das KRS 1 eingeplant sind. 
Methode 3.0: Datengrundlage aus dem Forschungsprojekt GLASSHOUSE: Um einen Einordnungsrahmen zu ermöglichen, einen Vergleich der Ergebnisse aufzustellen und daraus eine Handlungsempfehlung zu formulieren, wird zunächst die Ausgangslage bzw. die Lagerstruktur der jeweiligen KRS erläutert (vgl. Tabelle $10)$.

Tabelle 10. Beschreibung der Kommissionierszenarien (KRS)

\begin{tabular}{|c|c|}
\hline$K R S$ & Beschreibung des Kommessionierszenarios \\
\hline \#1 & $\begin{array}{l}\text { Vollpalettenkommissionierung, Block- und Hochregallager, zwei Smart Glasses: } \\
\text { Bei Vollpaletten handelt es sich um artikelreine Paletten, die in dieser Form vom Lie- } \\
\text { feranten erstellt und an nachgelagerte Empfänger weitergereicht werden. Hier wird } \\
\text { auch von Entnahme aus Originalpaletten gesprochen. Diese sind in Form von Block- } \\
\text { lägern gelagert, welche in gestapelter und ungestapelter Form ausgerichtet sein kön- } \\
\text { nen. In der Regel wird ein Lagerplatz pro Blocklager vergeben. Es gibt verschiedene } \\
\text { Regalarten, bei denen zusätzliche Transportmittel oder Personen eingesetzt werden } \\
\text { können, um Ein- und Auslagerungsvorgänge durchzuführen. Das Lagersystem be- } \\
\text { steht aus Lagergut, Lager- und Transporttechnik sowie einem Lagerverwaltungssys- } \\
\text { tem. Ein Hochregallager umfasst i. d. R. eine Regalhöhe von zwölf bis } 50 \text { Metern, bis } \\
\text { zu 100.000 Paletten- und entsprechend mehrere } 100.000 \text { Behälterplätze (Hompel } \\
\text { 2011). }\end{array}$ \\
\hline \#2 & $\begin{array}{l}\text { Einstufige Kleinteilkommissionierung, e-Commerce, sieben Smart Glasses: } \\
\text { Die einstufige Kommissionierung umfasst eine auftragsorientierte Kommissionie- } \\
\text { rung, bei der alle zu packenden Artikel in einem Auftrag nacheinander zusammenge- } \\
\text { stellt werden. Durch das e-Commerce wird eine elektronische Zustellung von Infor- } \\
\text { mationen über das Internet gewährleistet, bspw. in Form von Kauf und Verkauf von } \\
\text { Waren über einen Online-Shop. Außerdem wird oftmals eine elektronische Kommu- } \\
\text { nikation angestrebt. Die Kleinteilkommissionierung bzw. -lagerung zeichnet sich in } \\
\text { der Regel durch Kleinbehälter aus, welche die Funktionen eines Ladungsträgers er- } \\
\text { füllen. Sie umfassen den Lagerbedarf in Behälterregale und dienen als Transportmit- } \\
\text { tel für einen Verladeprozess (Martin 2011). }\end{array}$ \\
\hline \#3 & $\begin{array}{l}\text { Zweistufige Kleinteilkommissionierung, e-Commerce, } 20 \text { Smart Glasses im Einsatz: } \\
\text { Die zwei- oder mehrstufige Kommissionierung ist artikelorientiert, sodass zunächst } \\
\text { mehrere Aufträge gesammelt werden. Im Kommissionierungsprozess werden die Ar- } \\
\text { tikel nacheinander den jeweiligen Aufträgen zugeordnet und eine gleichzeitige Bear- } \\
\text { beitung von mehreren Aufträgen wird gewährleistet. Die mehrstufige Kommissionie- } \\
\text { rung wird oftmals mit dem Multi-order-Management in Verbindung gebracht. Der } \\
\text { daraus resultierende Vorteil durch den Einsatz der Smart Glasses in mehrstufigen } \\
\text { Prozessen ist zum einem der softwaretechnische Support für diese Prozesse, der } \\
\text { durch die Smart Glasses gewährleistet ist. Zum anderen steht weniger die Führung } \\
\text { der Mitarbeiter durch das Lager als vielmehr die Übermittlung von Entnahmeinfor- } \\
\text { mationen im Fokus (ten Hompel, Michael, Sadowsky, Volker, Beck 2011). }\end{array}$ \\
\hline
\end{tabular}

Nachfolgend werden die Inhalte des Angebotsvergleichs aufgezeigt und mit Hilfe der jeweils errechneten Kennzahlen bewertet. Die repräsentativen KRS und der Angebotsvergleich stellen somit einen Einordnungsrahmen für Unternehmen und deren individuell ermittelten betriebswirtschaftlichen Kennzahlen dar. Für das Endergebnis besteht die Möglichkeit, eine Zusammenfassung des Returns, der re- 
duzierten FQ und der erhöhten AG durchzuführen. Dadurch lassen sich die Effizienz- und Kostentreiber gegenüberstellen und bewerten (vgl. Tabelle 11).

Tabelle 11. Angebotsvergleich und Kennzahlen

\begin{tabular}{|c|c|c|c|}
\hline Kommissionierszenarien & $\# 1$ & $\# 2$ & $\# 3$ \\
\hline Anzahl Smart Glasses & 2 & 7 & 20 \\
\hline $\begin{array}{l}\text { Return - time-saving } \\
\text { (MTM-Analyse) }\end{array}$ & $35.334,00 €$ & $98.918,40 €$ & $141.312,00 €$ \\
\hline \multicolumn{4}{|l|}{ Angebot 1} \\
\hline Einm. Kosten - Rabatt & $37.500,00 €$ & $54.500,00 €$ & $99.500,00 €$ \\
\hline jährliche/fixe Kosten & $2.170,00 €$ & $2.870,00 €$ & $4.690,00 €$ \\
\hline Gesamtkosten (ein Jahr) & $39.670,00 €$ & $57.370,00 €$ & $104.190,00 €$ \\
\hline Gesamtkosten:(5 Jahre) & $48.350,00 €$ & $68.850,00 €$ & $122.950,00 €$ \\
\hline Anteil Hardwarekosten & $16,87 \%$ & $39,51 \%$ & $60,83 \%$ \\
\hline Integrationskosten & $4.200,00 € / \mathrm{Tag}$ & $4.200,00 € / \mathrm{Tag}$ & $4.200,00 € / \mathrm{Tag}$ \\
\hline $\begin{array}{l}\text { Lizenzkosten (pro Be- } \\
\text { trieb) }\end{array}$ & $14.800,00 €$ & $14.800,00 €$ & $14.800,00 €$ \\
\hline ROI & $0,89=89 \%$ & $1,72=172 \%$ & $1,36=136 \%$ \\
\hline Amortisationsdauer & 1,12 Jahre & 0,58 Jahre & 0,73 Jahre \\
\hline \multicolumn{4}{|l|}{ Angebot 2} \\
\hline Einm. Kosten - Rabatt & $35.620,00 €$ & $43.370,00 €$ & $63.520,00 €$ \\
\hline jährliche/fixe Kosten & $800,00 €$ & $2.800,00 €$ & $8.000,00 €$ \\
\hline Gesamtkosten (ein Jahr) & $36.420,00 €$ & $46.170,00 €$ & $71.520,00 €$ \\
\hline Gesamtkosten:(5 Jahre) & $39.620,00 €$ & $57.370,00 €$ & $103.520,00 €$ \\
\hline Anteil Hardwarekosten & $10,67 \%$ & $30,67 \%$ & $59,82 \%$ \\
\hline Integrationskosten & $16.560,00 €$ & $16.560,00 €$ & $16.560,00 €$ \\
\hline Lizenzkosten & $(400,00 €$ pro $S G)$ & $(400,00 €$ pro $\mathrm{SG})$ & $(400,00 €$ pro $\mathrm{SG})$ \\
\hline (pro Betriebsstätte) & $800,00 €$ & $2.800,00 €$ & $8.000,00 €$ \\
\hline ROI & $0,97=97 \%$ & $2,14=214 \%$ & $1,98=198 \%$ \\
\hline Amortisationsdauer & 1,12 Jahre & 0,58 Jahre & 0,73 Jahre \\
\hline \multicolumn{4}{|l|}{ Angebot 3} \\
\hline Einm. Kosten - Rabatt & $49.308,00 €$ & $54.558,00 €$ & $68.208,00 €$ \\
\hline jährliche/fixe Kosten & $5.400,00 €$ & $6.900,00 €$ & $10.800,00 €$ \\
\hline Gesamtkosten (ein Jahr) & $54.708,00 €$ & $61.458,00 €$ & $79.008,00 €$ \\
\hline Gesamtkosten:(5 Jahre) & $76.308,00 €$ & $89.058,00 €$ & $122.208,00 €$ \\
\hline Anteil Hardwarekosten & $5,79 \%$ & $18,41 \%$ & $42,44 \%$ \\
\hline Integrationskosten & ca. $19.200,00 €$ & ca. $19.200,00 €$ & ca. $19.200,00 €$ \\
\hline Lizenzkosten & $5.900,00 €$ & $5.900,00 €$ & $5.900,00 €$ \\
\hline ROI & $0,64=64 \%$ & $1,61=161 \%$ & $1,79=179 \%$ \\
\hline Amortisationsdauer & 2,16 Jahre & 0,90 Jahre & 0,86 Jahre \\
\hline
\end{tabular}

Aus der Zusammenstellung der Ergebnisse lässt sich schlussfolgern, dass mit dem Einsatz von Smart-Glasses-basierten Assistenzsystemen in den aufgezeigten Anwendungsfällen mit einer Amortisierung von $<1 \mathrm{Jahr}$ und einem positiven ROI $\mathrm{zu}$ rechnen ist. Außerdem ist ein effizienter Einsatz stark von der Anzahl der eingesetzten Endgeräte abhängig, da hier sowohl Kostentreiber als auch der Return stark variieren können. Es empfiehlt sich also die betriebsinterne Kommissionier- 
situation zu definieren und den damit zusammenhängenden Digitalisierungsgrad zu identifizieren. Daraufhin sollte ein Angebotsvergleich durchgeführt werden, da die Kosten zwischen den Anbietern variieren. Als Beispiel kann im vorliegenden Fall eine Verdopplung der Amortisationsdauer bei einem Vergleich von Angebot 1 und Angebot 3 für KRS 1 beobachtet werden.

\section{Implikation für die Praxis: Benchmark}

In der heutigen Zeit wird der Begriff „Benchmark“ als Vergleichsgröße interpretiert, die eine gemessene und erreichbare Leistung auf einem bestimmten Gebiet oder Segment darstellt und die systematische Suche nach besseren Lösungen innerhalb der eigenen Branche kennzeichnet (Siebert und Kempf 1998). In der Betriebswirtschaftslehre wird der Benchmark als Managementinstrument verwendet, um einen Marktvergleich zu erstellen (Capone 2007). Im Rahmen dieses Beitrags werden finanzwirtschaftliche Kennzahlen, Treiber der Wirtschaftlichkeit und Referenzwerte in Bezug auf unterschiedliche Kommissionierungsverfahren miteinander verglichen. Dadurch wird ein Vergleich der Effizienz des betriebsinternen Einsatzes von Smart-Glasses-basierten Lösungen in der Kommissionierung zu Mitbewerbern am Markt ermöglicht. In erster Linie wird dabei der Aspekt der Kostenreduktion einer technischen Lösung betrachtet (Siebert und Kempf 1998).

Um einen Benchmark durchführen zu können, werden in einer Vorbereitungsphase die gewünschten Eigenschaften der zu vergleichenden Daten vereinheitlicht. Hierfür können bspw. die Kennzahlen ROI und Amortisationsdauer von MDE-Geräten und Smart Glasses miteinander verglichen werden. Die Effizienztreiber sollten aus gleichen Quellen stammen und somit aufgrund einer einheitlichen Basis ermittelt worden sein. In der darauffolgenden Auswahlphase findet der tatsächliche Vergleich der Parameter statt. Hierfür werden die ermittelten Effizienz- und Kostentreiber einander gegenübergestellt.

Während in diesem Beitrag eine quantitative Herleitung von Kennzahlen vorgeführt wurde, zeigt ein Gerätevergleich deutlich auf, dass die qualitativen Faktoren ebenfalls essentiell Unterschiede aufweisen. Für Unternehmen in der Praxis werden in diesem Beitrag also notwendige Vergleichswerte für die Investitionskosten, Zeitersparnisse, Beschleunigung der AG als auch Reduzierung der FQ und den resultierenden Kennzahlen ROI und Amortisationsdauer für drei ausgewählte KRS gegeben, um einen unternehmensindividuellen Benchmark durchzuführen. Somit formuliert dieser Beitrag eine weitere Implikation für die Praxis.

\section{Zusammenfassung und Diskussion}

Mit Hilfe einer systematischen Literaturrecherche in Bezug auf den prozessoptimierenden Einsatz von Smart Glasses in der Logistik wurden Effizienztreiber ermittelt, die zu finanziellen und zeitlichen Einsparungen führen können. Diese werden zum einen durch eine erhöhte Arbeitsgeschwindigkeit ermöglicht, wo- 
durch Zeiteinsparungen innerhalb von Geschäftsprozessen monetär bewertet werden können. Durch Automatisierung bzw. Wegfall von Teilprozessen oder Beschleunigung von Laufwegen steigt die Pickleistung von Kommissionierern, wodurch eine erhöhte Umsatzgenerierung realisiert wird. Ein solches Szenario wurde mithilfe der MTM-Analyse an drei repräsentativen KRS angewendet und beispielhaft für das KRS 1 dargestellt.

Zum anderen fällt die reduzierte Fehlerquote unter die Treiber der Wirtschaftlichkeit von Smart Glasses, da Fehlpicks in der Kommissionierung teilweise hohe Kosten verursachen können. Mithilfe der Prozessführung und des Informationszugriffes durch die Smart Glasses, können Fehlgriffe vermieden werden. Wie sich eine Einsparung durch Vermeidung von Fehlern ermitteln lässt, wurde beispielhaft in Tabelle 9 aufgezeigt.

Damit ein Unternehmer abschätzen kann, ob die Investition in Smart Glasses für den eigenen Betrieb wirtschaftlich ist, kann ein brancheninterner Vergleich von unterschiedlichen Smart-Glasses-Anbietern auf Basis unterschiedlicher KRS als Hilfestellung herangezogen werden (Angebotsvergleich). Hierfür kann ein Benchmark mithilfe finanzwirtschaftlicher Kennzahlen wie dem ROI oder der Amortisationsdauer durchgeführt werden. Die KRS stellen markttypische Lagerstrukturen dar, wodurch Unternehmen ein Einordnungsrahmen für eigene Kommissioniersituationen gegeben wird. Festzustellen ist, dass sowohl der ROI als auch die Amortisationsdauer stark von der Anzahl der eingesetzten Smart Glasses abhängig ist. Dabei konnte unter Betrachtung der Angebote der unterschiedlichen Smart-Glasses-Anbieter unterschiedliche Investitionsgrößen bzw. Kostentreiber ermittelt werden.

Die Literaturrecherche hat außerdem ergeben, dass nur verhältnismäßig wenig Quellen repräsentative Ergebnisse in Form von verwertbaren Effizienztreiber aufzeigen. Oftmals basieren diese auf Labortests und sind somit nicht vergleichbar mit realen Situationen in der Praxis. Als Konsequenz wurde eine umfangreiche Anbieterrecherche durchgeführt, um die Resultate der Literaturrecherche zu verifizieren.

Der Beitrag liefert darüber hinaus eine Auswahl an Methoden, welche zur Bewertung der Wirtschaftlichkeit verwendet werden können. Die Erweiterung der WPM-Methode um eine Zeiteinheitsanalyse von dargestellten Prozessen in Form einer MTM-Analyse orientiert sich an einer individuellen Anwendung in der Praxis. Für den Fall, dass die praktische Umsetzung einer WPM-Methode etwa durch unzureichende Daten zur Darstellung eines Ist-Prozesses verhindert wird, ermöglicht das Verwenden von alternativen Methoden trotzdem eine Wirtschaftlichkeitsbewertung.

\section{Ausblick}

Die Einführung von Smart Glasses im Zuge der Industrie 4.0 ermöglicht einen wesentlichen Beitrag zur Digitalisierung von Arbeitsprozessen in der Logistik. 
Durch angewandte Methoden und eine umfangreiche Recherche lässt sich feststellen, dass auch ein positiver Wirtschaftlichkeitsgrad erwartet werden kann.

Aufgrund der Betrachtung von repräsentativen Kommissionierszenarien in diesem Beitrag lässt sich vermuten, dass bei niedrigerem Digitalisierungsgrad zwar mit einer erschwerten Integration von innovativen Technologien zu rechnen ist, der Grad der Wirtschaftlichkeit zugehöriger Wertschöpfungsprozesse jedoch ansteigt. Als Konsequenz kann die Einführung von Smart-Glasses-basierten Assistenzsystemen sowohl für Groß- als auch klein- und mittelständische Unternehmen als eine wirtschaftliche Digitalisierungsmaßnahme angesehen werden.

Eine Betrachtung der aktuellen Forschung hat ergeben, dass eine branchenübergreifende Skalierung von AR- oder Smart-Glasses-basierten Assistenzsystemen aufgrund einer mangelnden Datengrundlage zwar vermutet, jedoch noch nicht bestätigt werden kann. Die Anzahl von AR-Technologien, die den Marktzugang finden, steigt stetig. Jedoch fehlt es noch an ausreichend Installationen in Industrieunternehmen. Ebenfalls ist ein Mangel an Langzeitbeobachtungen festzustellen, welcher wie bei nahezu allen innovativen Technologien eine umfassende Wirtschaftlichkeitsbewertung verhindert. Dieser Beitrag stellt besonders in diesem $\mathrm{Zu}$ sammenhang einen Mehrwert für die Praxis dar, um eine Vorstellung für das Potenzial der AR-Technologie im logistischen Einsatz zu bekommen. Die Ergebnisse leiten dazu ab, dass die Anzahl an Realinstallation in den kommenden Jahren ansteigen könnte. Aufgrund der sich wiederholenden Tätigkeiten in der Logistik, dem hohem Informationsbedarf als auch dem Wunsch, beide Hände aktiv im Arbeitsprozess zu verwenden, ist anzunehmen, dass der zugehörige Branchenzweig eine Vielzahl an potenziellen Anwendungsfällen bereitstellt. Als Konsequenz liefert die Logistikbranche eine Vielzahl an geeigneten Praktiken und konkreten Lösungsansätzen für die Anwendung von Smart Glasses. Diese können in andere Branchen adaptiert werden und sorgen somit für wachsende branchenübergreifende Verfügbarkeit von Smart-Glasses-basierten Assistenzsystemen.

\section{Literatur}

Almeida DLM de, Ferreira JCE (2009) Analysis of the Methods Time Measurement (MTM) Methodology through its Application in Manufacturing Companies. In: Flex Automation Intelligent Manufactoring. FAIM, United Kingdom, Middlesbrough

Arnold D, Furmans K, Isermann H, Kuhn A, Tempelmeier H (2008) Handbuch Logistik. Springer, Berlin, Heidelberg

Becker J, Kugeler M, Rosemann M (2012) Prozessmanagement. Gabler Verlag, Wiesbaden Benmoussa R, Charkaoui A, Abd A, Hassou M (2015) Capability/maturity based model for logistics processes assessment: Application to distribution processes. International Journal of Productivity and Performance Management 64(1):28-51. doi: 10.1108/IJPPM-08-2012-0084.

Böning C, Kasselmann S (2018) Schlussbericht - Entwicklung eines Reifegradmodells zur Steigerung der Industrie 4.0 - Befähigung in Produktion und Logistik am Beispiel von 
interaktiven Assistenzsystemen (Ready 4.0). Industrielle Gemeinschaftsforschung, Forschungsnetzwerk Mittelstand, Hannover

Capone R (2011) Kostenrechnung für Elektrotechniker. Vieweg+Teubner Verlag, Wiesbaden. doi:10.1007/978-3-8348-8104-5

Cinovation (2018) Pick-by-Vision.

https://www.cinovation.de/web/images/cinovation/flyer/CINOVATION_PickByVisio n2018DE.pdf, abgerufen am 07.07.2020

Crostack HA, Schlüter N, Mathis J (2008) Simulation zur Qualitäts- und Kostenbewertung bei manueller Kommissionierung. In: Rabe M (Hrsg) Advances in Simulation for Production and Logistics Applications. Fraunhofer-IRB-Verlag, Stuttgart

Dataphone (2017) Neue Innovationsfelder durch Smart Glasses und Customer Devices, https://lineapro.net/media/files/DIWA_SmartGlasses_GS1ForumIntralogistics2017.pdf , abgerufen am 07.07.2020

De Vries J, De Koster R, Stam D (2016) Exploring the role of picker personality in predicting picking performance with pick by voice, pick to light and RF-terminal picking. International Journal of Production Research 54(8):2260-2274. doi: 10.1080/00207543.2015.1064184.

Dellmann K, Pedell KL (1994) Controlling von Produktivität, Wirtschaftlichkeit und Ergebnis, Berichte aus der Arbeit der Schmalenbach-Gesellschaft. Schäffer-Poeschel Verlag, Stuttgart

Deutsche Post DHL Group (2015) https://www.dpdhl.com/de/presse/pressemitteilungen/2015/dhl-testet-augmentedreality-anwendung.html, abgerufen am 13.07.2020

Eichhorn P, Merk J (2016) Das Prinzip Wirtschaftlichkeit. Gabler Verlag, Wiesbaden. doi:10.1007/978-3-658-07830-0

Furmans K, Linsel P (2011) Leichtbau bei Unstetigkeitförderern durch Einsatz moderner Werkstoffe. Logistics Journal, Proceedings, Online Journal. doi:10.2195/LJ_proc_furmans_de_201108_01

Galka S, Ulbrich A (2008) Kommissioniersysteme. Präsentation, Technische Universität München, https://docplayer.org/60850371-Kommissioniersysteme-bausteine-fuer-diesimulation-von-kommissioniersystemen.html, abgerufen am 13.07.2020

Gleißner H, Femerling C (2008) Logistik. Gabler, Wiesbaden

Günthner WA (2010) Augmented Reality supported Order Picking using Projected User Interfaces. ACM Conference Computer Human Interface, (131), 1695-1704

Günthner WA, Blomeyer N, Reif R, Schedlbauer M (2009) Pick-by-Vision: Augmented Reality unterstützte Kommissionierung. In: Günthner WA (Hrsg) fml - Lehrstuhl für Fördertechnik Materialfluss Logistik. Technische Universität München

Guo A, Starner T, Raghu S, Xie X, Ismail S, Luo X, Simoneau J, Gilliland S, Baumann H, Southern C (2014) A comparison of order picking assisted by head-up display (HUD), cart-mounted display (CMD), light, and paper pick list. In: Proceedings of the 2014 ACM International Symposium on Wearable Computers (ISWC '14), Association for Computing Machinery, New York. doi: 10.1145/2634317.2634321

Hausladen I (2016) IT-gestützte Logistik. Gabler Verlag, Wiesbaden

Henderson J (2020) Geodis begins smart glasses initiative in Cologne facility, Digital Supply Chain, https://www.supplychaindigital.com/technology/geodis-begins-smartglasses-initiative-cologne-facility, abgerufen am 07.07.2020

Herter J (2014) Augmented Reality supported Order Picking using Projected User Interfaces. Bachelorarbeit, Universität Stuttgart 
Kaplan RS, Norton DP (2000) The Strategy-Focused Organization - How Balanced Scorecard Companies Thrive in the New Business Environment. Harvard Business Review Press, Brighton

Loske D, Kaspar B (2018) Eignungsbeurteilung eines Augmented Reality basierten Kommissioniersystems für den Einsatz in der Lagerlogistik des Lebensmitteleinzelhandels. Institut für Logistik- \& Dienstleistungsmanagement (ild) FOM Hochschule, Essen

Ludwig C, Reimann C (2005) Augmented Reality: Information im Fokus. C-LAB Report Cooperative Computing \& Communication Laboratory 4(1):4-15

Martin H (2011) Kommissioniersysteme. In: Martin H (Hrsg) Transport- und Lagerlogistik. Vieweg+Teubner Verlag, Wiesbaden, 8. Auflage

Matt C, Hess T, Benlian A (2015) Digital Transformation Strategies. Bus Inf Syst Eng 57:339-343

Mieg, H, Brunner, B (2001) Experteninterviews - Eine Einführung und Anleitung. MUB Working Paper 6, ETH Zürich, Professur für Mensch-Umwelt-Beziehungen, https://doi.org/10.3929/ethz-a-004218020

O’Regan G (2014) Introduction to Software Quality. Springer International Publishing, Basel

Picavi (2016a) Vorteile des Einsatzes von Datenbrillen in der Kommissionierung in Verbindung mit der Picavi-Systemlösung. https://www.doag.org/formes/pubfiles/9141933/2017-L_D-Dirk_FrankeKommissionieren_mit_Datenbrille_Laeuft_Echt_Jetzt_-Praesentation.pdf, abgerufen am 13.07.2020

Picavi (2016b) Klosterfrau. https://picavi.com/case-study/klosterfrau-healthcare-group/, abgerufen am 07.07.2020

Picavi (2017) Fiege. https://picavi.com/case-study/fiege/, abgerufen am 07.07.2020

Picot A, Reichwald R (1984) Bürokommunikation. Leitsätze für den Anwender.

Reif R (2009) Entwicklung und Evaluierung eines Augmented Reality unterstützten Kommissioniersystems. Dissertation, Technische Universität München

Reif R, Günthner WA (2009) Pick-by-vision: augmented reality supported order picking. The Visual Computer 25:461-467. doi:10.1007/s00371-009-0348-y

Reif R, Günthner WA, Schwerdtfeger B, Klinker G (2009) Pick-by-Vision Comes on Age: Evaluation of an Augmented Reality Supported Picking System in a Real Storage Environment. In: Proceedings of the 6th International Conference on Computer Graphics, Virtual Reality, Visualisation and Interaction in Africa. Association for Computing Machinery, New York, doi:10.1145/1503454.1503459

Richter NB (2018) Einführung einer scannergeführten beleglosen Kommissionierung am Imperial Standort Langelsheim. Bachelorarbeit, Ostfalia Hochschule für angewandte Wissenschaften, Wolfenbüttel

Scheer AW, Nüttgens M, Zimmermann V (1997) Objektorientierte Ereignisgesteuerte Prozeßkette (oEPK) - Methode und Anwendung. Institut für Wirtschaftsinformatik (IWi) an der Universität des Saarlandes, Saarbrücken 141:1-23

Siebert G, Kempf S (1998) Mit Benchmarking die Prozesse optimieren. In: Qualität und Zuverlässigkeit. Carl Hanser Verlag, München

Sommerhäuser L (2017) Was Handhelds leisten müssen. https://www.it-zoom.de/mobilebusiness/e/was-handhelds-leisten-muessen-17089/, abgerufen am 07.07.2020

Stoll J (2015) A decomposition approach for the analysis of discrete- time queuing networks with finite buffers. In: Görlitz R, Bertsch V, Caton S, Feldmann N, Jochem P, 
Maleshkova M, Reuter-Oppermann M (Eds) Proceedings of the First Karlsruhe Service Summit - Advances in Service Research. KIT Scientific Publishing, Karlsruhe

ten Hompel M, Sadowsky V, Beck M (2011) Kommissionierung. Springer Verlag, Berlin Heidelberg, doi:10.1007/978-3-540-29940-0

ten Hompel M, Schmidt T (2010) Warehouse Management. Springer Verlag, Berlin Heidelberg, doi:10.1007/978-3-642-03185-4

Ternès A, Schieke S (2018) Mittelstand 4.0. Springer Gabler, Wiesbaden. doi:10.1007/9783-658-20917-9

Tompkins J, White J, Bozer Y, Tanchoco J (2010) Facilities Planning. John Wiley \& Sons, New Jersey

Ubimax (2014a) DHL-Referenz. https://www.ubimax.com/case-study/dhl-2016, abgerufen am 13.07.2020

Ubimax (2014b) WS Systems. https://mk0ubimaxcomowq7qxky.kinstacdn.com/wpcontent/uploads/2020/04/customer-projects-20180502-1-WS-Kunststoff.pdf, abgerufen am 13.07.2020

Ubimax (2015) DHL 2015. https://www.ubimax.com/case-study/dhl-2015, abgerufen am 13.07.2020

Ubimax (2015a) DHL Smart glasses as new standard in logistics,. https://www.ubimax.com/en/references/dhl-casestudy-2017.html, abgerufen am 07.07.2020

Ubimax (2015b) Schnellecke. https://mk0ubimaxcomowq7qxky.kinstacdn.com/wpcontent/uploads/2020/04/customer-projects-20180504-Schnellecke.pdf, abgerufen am 13.07.2020

Ubimax (2017a) Case Studies Ubimax. https://www.ubimax.com/en/references/\#casestudies, abgerufen am 07.07.2020

Ubimax (2017b) Intel. https://mk0ubimaxcomowq7qxky.kinstacdn.com/wpcontent/uploads/2020/04/customer-projects-Intel.pdf, abgerufen am 13.07.2020

Ubimax (2018) Angabe im Rahmen eines Experteninterviews mit Ubimax im GLASSHOUSE-Projekt

Upskill G (2017) Upskill and GE. https://upskill.io/landing/upskill-and-ge/, abgerufen am 13.07.2020

Weaver K, Baumann H, Starner T, Iben H, Lawo M (2010) An Empirical Task Analysis of Warehouse Order Picking Using Head-Mounted Displays. In: Proceedings of the SIGCHI Conference on Human Factors in Computing Systems. Association for Computing Machinery, New York. doi:10.1145/1753326.1753580.

Webster J, Watson RT (2002) Analyzing the Past to Prepare for the Future: Writing a Literature Review. MIS Quarterly 26(2):xiii-xxiii

Xpert.digital (2016) Was Fehlpicks kosten. https://xpert.digital/was-fehlpicks-kosten/, abgerufen am 07.07.2020

Zilker M (2001) Automatisierung unscharfer Bewertungsverfahren - Modellierung und prototypische Umsetzung am Beispiel von Virtual Reality Projekten. Dissertation, Technische Universität Dresden 


\section{Anhang A - Ergänzung der Effizienztreiber aus Literaturrecherche}

\begin{tabular}{|c|c|c|c|c|c|c|c|c|c|}
\hline $\begin{array}{l}\text { Legende: } 1 . \\
\text { Fehlerquote } \\
\text { Produktiviti }\end{array}$ & $\begin{array}{l}\text { Prod } \\
\text { enred } \\
\text { ätsste }\end{array}$ & $\begin{array}{l}\text { duktivitätsst } \\
\text { uzierung, } 4 \\
\text { igerung Pic }\end{array}$ & $\begin{array}{l}\text { teigerur } \\
\text { 1. Fehle } \\
\text { ck-by-V }\end{array}$ & $\begin{array}{l}\text { ng gg. } \\
\text { rquote } \\
\text { ision }\end{array}$ & $\begin{array}{l}\text { Pick-l } \\
\text { pro } P \\
\text { gg. Pic }\end{array}$ & $\begin{array}{l}\text { y-Scan, } 2 . \\
\text { ck, 5. Dau } \\
k \text {-by-Liste, }\end{array}$ & $\begin{array}{l}\text { Produk } \\
\text { ıer, 6. } Q \\
\text { 9. Rech }\end{array}$ & $\begin{array}{l}\text { tivitätssteigerung gg. Pick } \\
\text { uelle, 7. Praxis/Labortest } \\
\text { henweg Fehlerquotenredu }\end{array}$ & $\begin{array}{l}\text {-by-List/Paper, } 3 . \\
\text { 8. Rechenweg } \\
\text { zierung }\end{array}$ \\
\hline & 1. & 2. & 3. & 4. & 5. & 6. & 7. & 8. & 9. \\
\hline $\begin{array}{l}\text { Pick-by- } \\
\text { Voice }\end{array}$ & --- & $10 \%$ & -- & -- & -- & $\begin{array}{l}\text { Loske \& } \\
\text { Kaspar } \\
(2018)\end{array}$ & Praxis & nur genannt & - \\
\hline $\begin{array}{l}\text { Pick-by- } \\
\text { Vision }\end{array}$ & --- & $19 \%$ & $67 \%$ & -- & -- & $\begin{array}{l}\text { Loske \& } \\
\text { Kaspar } \\
(2018)\end{array}$ & Praxis & nur genannt & - \\
\hline $\begin{array}{c}\text { Fallstudie } 2 \\
\text { Pick- by- } \\
\text { Vison }\end{array}$ & $\begin{array}{c}8-12 \\
\%\end{array}$ & -- & -- & -- & -- & $\begin{array}{l}\text { Loske \& } \\
\text { Kaspar } \\
(2018)\end{array}$ & Praxis & nur genannt & - \\
\hline $\begin{array}{l}\text { Pick-by- } \\
\text { Paper }\end{array}$ & --- & --- & --- & $\begin{array}{c}0.562 \\
5 \%\end{array}$ & $\begin{array}{c}07: 0 \\
6,8\end{array}$ & $\begin{array}{l}\text { Herter } \\
(2014)\end{array}$ & $\begin{array}{l}\text { Labor- } \\
\text { tests }\end{array}$ & $\begin{array}{c}00: 07: 07=100 \%= \\
426 \mathrm{sec}\end{array}$ & genannt \\
\hline $\begin{array}{l}\text { Pick-by- } \\
\text { Vision }\end{array}$ & --- & $\begin{array}{l}\text { Produktivi- } \\
\text { tätsminde- } \\
\text { rung um } \\
115 \%\end{array}$ & $\begin{array}{c}0.437 \\
5 \%\end{array}$ & $\begin{array}{c}0.125 \\
\%\end{array}$ & $\begin{array}{c}15: 1 \\
8,7\end{array}$ & $\begin{array}{l}\text { Herter } \\
(2014)\end{array}$ & $\begin{array}{l}\text { Labor- } \\
\text { tests }\end{array}$ & $\begin{array}{l}426 \mathrm{sec} / 100 * 215=916 \\
->(215-100 \%=115 \%)\end{array}$ & $\begin{array}{c}0,5625-0,125= \\
0,4375\end{array}$ \\
\hline $\begin{array}{l}\text { Order- } \\
\text { PickAR }\end{array}$ & --- & $50 \%$ & $\begin{array}{c}\text { FQ- } \\
\text { Stei- } \\
\text { ge- } \\
\text { rung } \\
0,125 \\
\%\end{array}$ & $\begin{array}{c}0.687 \\
5 \%\end{array}$ & $\begin{array}{c}03: 3 \\
3,6\end{array}$ & $\begin{array}{l}\text { Herter } \\
(2014)\end{array}$ & $\begin{array}{l}\text { Labor- } \\
\text { tests }\end{array}$ & $\begin{array}{c}426 \mathrm{sec} \\
/ 100 * 50=213(213 \mathrm{sec}=3: 3 \\
3 \min )->(50 \%)\end{array}$ & $\begin{array}{c}0,5625-0,6875= \\
-0,125\end{array}$ \\
\hline $\begin{array}{l}\text { Pick-by- } \\
\text { Voice }\end{array}$ & --- & $4,22 \%$ & $\begin{array}{c}\text { FQ- } \\
\text { Stei- } \\
\text { ge- } \\
\text { rung } \\
0,687 \\
5 \%\end{array}$ & $\begin{array}{c}1.25 \\
\%\end{array}$ & $\begin{array}{c}06: 4 \\
8,8\end{array}$ & $\begin{array}{l}\text { Herter } \\
(2014)\end{array}$ & $\begin{array}{l}\text { Labor- } \\
\text { tests }\end{array}$ & $\begin{array}{c}426 \mathrm{sec} \\
/ 100 * 95,7=408(408 \mathrm{sec}=6 \\
: 48 \mathrm{~min})->(4,22 \%)\end{array}$ & $\begin{array}{c}0,5625-1,25=- \\
0,6875\end{array}$ \\
\hline
\end{tabular}

\begin{tabular}{|c|c|c|c|c|c|c|c|c|}
\hline $\begin{array}{l}\text { Head-Up } \\
\text { Display } \\
\text { (HUD) }\end{array}$ & & $37 \%$ & $\begin{array}{c}2,25 \\
\%\end{array}$ & $\begin{array}{c}0,007 \\
5 \%: 38 \\
5 \%\end{array}$ & $\begin{array}{l}\text { Guo et al. } \\
\text { (2014) }\end{array}$ & $\begin{array}{c}\text { Labor- } \\
\text { tests }\end{array}$ & $\begin{array}{c}3747 \mathrm{sec} \\
-/ 100 * 62,53=2337(2337 \mathrm{se} \\
\mathrm{c}=38,57 \mathrm{~min})->(37,47 \\
\%)\end{array}$ & $\begin{array}{c}0,03-0,0075= \\
0,0225(2,25 \%)\end{array}$ \\
\hline $\begin{array}{l}\text { Cart- } \\
\text { Mounted } \\
\text { Display } \\
(\mathrm{CMD})\end{array}$ & & $36 \%$ & $1,7 \%$ & $\begin{array}{cc}0,013 & 0: 39 \\
\% & 58\end{array}$ & $\begin{array}{l}\text { Guo et al. } \\
\text { (2014) }\end{array}$ & $\begin{array}{l}\text { Labor- } \\
\text { tests }\end{array}$ & $\begin{array}{c}3747 \mathrm{sec} \\
-/ 100 * 64,17=2398(2398 \mathrm{se} \\
\mathrm{c}=38,58 \mathrm{~min})->(35,83 \%)\end{array}$ & $\begin{array}{c}0,03- \\
0,013=0,017(1,7 \\
\%)\end{array}$ \\
\hline $\begin{array}{l}\text { Pick-by- } \\
\text { Light }\end{array}$ & & $28 \%$ & $\begin{array}{c}\text { FQ- } \\
\text { Stei- } \\
\text { ge- } \\
\text { rung } \\
0,75 \\
\%\end{array}$ & $\begin{array}{c}0,0370: 45 \\
5 \% \quad 50\end{array}$ & $\begin{array}{c}\text { Guo et al. } \\
\text { (2014) }\end{array}$ & $\begin{array}{c}\text { Labor- } \\
\text { tests }\end{array}$ & $\begin{array}{c}3747 \mathrm{sec} \\
-/ 100 * 72,25=2700(2700 \mathrm{se} \\
\mathrm{c}=45,50 \mathrm{~min})->(27,75 \\
\%)\end{array}$ & $\begin{array}{l}0,03-0,0375=- \\
0,0075(-0,75 \%)\end{array}$ \\
\hline $\begin{array}{l}\text { Pick-by- } \\
\text { Paper }\end{array}$ & & -- & -- & $\begin{array}{cc}0,03 & 0: 62 \\
\% & 27\end{array}$ & $\begin{array}{l}\text { Guo et al. } \\
\text { (2014) }\end{array}$ & $\begin{array}{l}\text { Labor- } \\
\text { tests }\end{array}$ & $\begin{array}{c}0: 62,27 \mathrm{~min}=100 \\
\%=3747 \mathrm{sec}\end{array}$ & genannt \\
\hline $\begin{array}{l}\text { Head-Up } \\
\text { Display }\end{array}$ & 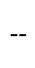 & $30 \%$ & $35 \%$ & $\begin{array}{cc}0,10 & 00: 4 \\
\% & 5\end{array}$ & $\begin{array}{l}\text { Weaver } \\
\text { et al. }\end{array}$ & $\begin{array}{l}\text { Labor- } \\
\text { tests }\end{array}$ & $\begin{array}{c}65 \mathrm{sec} \\
/ 100 * 69,23=45(45 \mathrm{sec})-\end{array}$ & $\begin{array}{c}0,45-0,10=0,35 \\
(35 \%)\end{array}$ \\
\hline
\end{tabular}


Legende: 1. Produktivitätssteigerung gg. Pick-by-Scan, 2. Produktivitätssteigerung gg. Pick-by-List/Paper, 3. Fehlerquotenreduzierung, 4. Fehlerquote pro Pick, 5. Dauer, 6. Quelle, 7. Praxis/Labortest, 8. Rechenweg Produktivitätssteigerung Pick-by-Vision gg. Pick-by-Liste, 9. Rechenweg Fehlerquotenreduzierung

\begin{tabular}{|c|c|c|c|c|c|c|c|c|}
\hline & 2. & 3. & 4. & 5. & 6. & 7. & 8. & 9. \\
\hline (HUD) & & & & & (2010) & & $>(30,77 \%)$ & \\
\hline $\begin{array}{l}\text { Pick-by- } \\
\text { Voice }\end{array}$ & $\begin{array}{c}\text { Produktivi- } \\
\text { tätsminde- } \\
\text { rung um } 15 \\
\%\end{array}$ & $17 \%$ & $\begin{array}{c}0,28 \\
\%\end{array}$ & $\begin{array}{c}01: 1 \\
5\end{array}$ & $\begin{array}{l}\text { Weaver } \\
\text { et al. } \\
(2010)\end{array}$ & $\begin{array}{l}\text { La- } \\
\text { bort- } \\
\text { ests }\end{array}$ & $\begin{array}{c}65 \mathrm{sec} \\
/ 100 * 115,38=75(75 \mathrm{sec})- \\
>(-15,38 \%)\end{array}$ & $\begin{array}{c}0,45-0,28=0,17 \\
(17 \%)\end{array}$ \\
\hline $\begin{array}{l}\text { Pick-by- } \\
\text { Paper } \\
\text { (grafisch) }\end{array}$ & $23 \%$ & $10 \%$ & $\begin{array}{c}0,35 \\
\%\end{array}$ & $\begin{array}{c}00: 5 \\
0\end{array}$ & $\begin{array}{l}\text { Weaver } \\
\text { et al. } \\
(2010)\end{array}$ & $\begin{array}{l}\text { La- } \\
\text { bort- } \\
\text { ests }\end{array}$ & $\begin{array}{c}65 \mathrm{sec} \\
/ 100 * 76,92=50(50 \mathrm{sec})- \\
>(23,08 \%)\end{array}$ & $\begin{array}{c}0,45-0,35=0,1 \\
(10 \%)\end{array}$ \\
\hline $\begin{array}{l}\text { Pick-by- } \\
\text { Paper } \\
\text { (Text) }\end{array}$ & --- & -- & $\begin{array}{l}0,45 \\
\%\end{array}$ & $\begin{array}{c}01: 0 \\
5\end{array}$ & $\begin{array}{l}\text { Weaver } \\
\text { et al. } \\
(2010)\end{array}$ & $\begin{array}{l}\text { La- } \\
\text { bort- } \\
\text { ests }\end{array}$ & $1: 05 \mathrm{~min}=100 \%=65 \mathrm{sec}$ & - \\
\hline $\begin{array}{l}\text { Pick-by- } \\
\text { Paper }\end{array}$ & ---- & $\begin{array}{l}\varnothing \mathrm{FQ} \\
0,35 \\
\% \\
\text { allg. }\end{array}$ & $\begin{array}{c}0.84 \\
\%\end{array}$ & $\begin{array}{c}29: 5 \\
4\end{array}$ & $\begin{array}{l}\text { Reif und } \\
\text { Günthner } \\
(2009)\end{array}$ & $\begin{array}{l}\text { Labor- } \\
\text { tests }\end{array}$ & - & genannt \\
\hline $\begin{array}{l}\text { Pick-by- } \\
\text { Vision }\end{array}$ & $\begin{array}{c}4 \% \text { beim } \\
\text { erstmaligen } \\
\text { Anwenden, } \\
\text { bei wieder- } \\
\text { holten Ma- } \\
\text { le } 19 \%\end{array}$ & $\begin{array}{c}0,72 \\
\%\end{array}$ & $\begin{array}{c}0.12 \\
\%\end{array}$ & $\begin{array}{c}28: 4 \\
6\end{array}$ & $\begin{array}{l}\text { Reif und } \\
\text { Günthner } \\
(2009)\end{array}$ & $\begin{array}{l}\text { Labor- } \\
\text { tests }\end{array}$ & genannt & $0,84-0,12=0,72 \%$ \\
\hline $\begin{array}{l}\text { Pick-by- } \\
\text { Voice }\end{array}$ & $\begin{array}{l}\text { Werte für die Fe } \\
\text { quote pro Pick w } \\
\text { dazugeschrieben }\end{array}$ & $\begin{array}{l}\text { ehler- } \\
\text { yurden } \\
\text { n, wo- }\end{array}$ & $\begin{array}{c}0.08 \\
\%\end{array}$ & -- & $\begin{array}{l}\text { Reif und } \\
\text { Günthner } \\
(2009)\end{array}$ & $\begin{array}{l}\text { Labor- } \\
\text { tests }\end{array}$ & -- & genannt \\
\hline $\begin{array}{l}\text { Pick-by- } \\
\text { Light }\end{array}$ & $\begin{array}{l}\text { bei nicht auf die } \\
\text { eingegangen } w \mathrm{l}\end{array}$ & $\begin{array}{l}\text { Quelle } \\
\text { urde }\end{array}$ & $\begin{array}{c}0.40 \\
\%\end{array}$ & -- & $\begin{array}{l}\text { Reif und } \\
\text { Günthner } \\
(2009)\end{array}$ & $\begin{array}{l}\text { Labor- } \\
\text { tests }\end{array}$ & -- & genannt \\
\hline $\begin{array}{l}\text { Pick-by- } \\
\text { Paper }\end{array}$ & -- & $\begin{array}{l}\varnothing \mathrm{FQ} \\
0,35 \\
\% \\
\text { allg. }\end{array}$ & $\begin{array}{c}0.84 \\
\%\end{array}$ & $\begin{array}{c}29: 5 \\
4\end{array}$ & $\begin{array}{l}\text { Reif et al. } \\
\text { (2009) }\end{array}$ & $\begin{array}{l}\text { Labor- } \\
\text { tests }\end{array}$ & -- & genannt \\
\hline $\begin{array}{l}\text { Pick-by- } \\
\text { Vision }\end{array}$ & $\begin{array}{l}4 \% \text { beim } \\
\text { erstmaligen } \\
\text { Anwenden, } \\
\text { bei wieder- } \\
\text { holten Ma- } \\
\text { le } 19 \%\end{array}$ & $\begin{array}{c}0,72 \\
\%\end{array}$ & $\begin{array}{c}0.12 \\
\%\end{array}$ & $\begin{array}{c}28: 4 \\
6\end{array}$ & $\begin{array}{l}\text { Reif et al. } \\
\text { (2009) }\end{array}$ & $\begin{array}{l}\text { Labor- } \\
\text { tests }\end{array}$ & genannt & $0,84-0,12=0,72 \%$ \\
\hline $\begin{array}{l}\text { Pick-by- } \\
\text { Voice }\end{array}$ & $\begin{array}{l}\text { Produktivi- } \\
\text { tätsminde- } \\
\text { rung um } 3 \\
\underset{\%}{0}\end{array}$ & $\begin{array}{c}\text { FQ- } \\
\text { Stei- } \\
\text { ge- } \\
\text { rung } \\
2,88 \\
\%\end{array}$ & $\begin{array}{c}4,94 \\
\%\end{array}$ & $\begin{array}{c}12,0 \\
7 \\
\min \end{array}$ & $\begin{array}{l}\text { Günthner } \\
\text { et al. } \\
(2009)\end{array}$ & $\begin{array}{l}\text { Labor- } \\
\text { tests }\end{array}$ & $\begin{array}{c}704 \\
/ 100 * 103=727(727 \mathrm{sec})- \\
>(-3 \%)\end{array}$ & $\begin{array}{c}2,06-4,94=-2,88 \\
\%\end{array}$ \\
\hline $\begin{array}{l}\text { Pick-by- } \\
\text { Vision }\end{array}$ & $4 \%$ & $\begin{array}{c}1,24 \\
\%\end{array}$ & $\begin{array}{c}0,82 \\
\%\end{array}$ & $\begin{array}{c}10,7 \\
6 \\
\min \end{array}$ & $\begin{array}{l}\text { Günthner } \\
\text { et al. } \\
(2009)\end{array}$ & $\begin{array}{l}\text { Labor- } \\
\text { tests }\end{array}$ & $\begin{array}{c}704 \\
/ 100 * 96,02=676(676 \mathrm{sec}) \\
->3,98 \%\end{array}$ & $2,06-0,82=1,24 \%$ \\
\hline Liste & & -- & $\begin{array}{c}2,06 \\
\%\end{array}$ & $\begin{array}{c}11,4 \\
4 \\
\min \end{array}$ & $\begin{array}{l}\text { Günthner } \\
\text { et al. } \\
(2009)\end{array}$ & $\begin{array}{l}\text { Labor- } \\
\text { tests }\end{array}$ & $\begin{array}{c}11,54 \min =100 \%= \\
704 \mathrm{sec}\end{array}$ & genannt \\
\hline $\begin{array}{l}\text { Pick-by- } \\
\text { Voice }\end{array}$ & \multicolumn{4}{|c|}{ nicht angegeben } & $\begin{array}{l}\text { Günthner } \\
\text { et al. }\end{array}$ & Praxis & - & - \\
\hline
\end{tabular}


Legende: 1. Produktivitätssteigerung gg. Pick-by-Scan, 2. Produktivitätssteigerung gg. Pick-by-List/Paper, 3. Fehlerquotenreduzierung, 4. Fehlerquote pro Pick, 5. Dauer, 6. Quelle, 7. Praxis/Labortest, 8. Rechenweg Produktivitätssteigerung Pick-by-Vision gg. Pick-by-Liste, 9. Rechenweg Fehlerquotenreduzierung

\begin{tabular}{|c|c|c|c|c|c|c|c|c|}
\hline 1 & 2. & 3. & 4. & 5. & 6. & 7. & 8. & 9. \\
\hline \multicolumn{9}{|c|}{ (2009) } \\
\hline $\begin{array}{l}\text { Pick-by- } \\
\text { Vision }\end{array}$ & $4 \%$ & $\begin{array}{c}0,72 \\
\%\end{array}$ & $\begin{array}{c}0,12 \\
\%\end{array}$ & $\begin{array}{c}28,4 \\
6 \\
\min \end{array}$ & $\begin{array}{l}\text { Günthner } \\
\text { et al. } \\
(2009)\end{array}$ & Praxis & $\begin{array}{c}1794 / 100 * 96,20=1726 \\
(1726 \mathrm{sec}=28,46 \mathrm{~min})- \\
>3,8 \%\end{array}$ & $0,84-0,12=0,72 \%$ \\
\hline Liste & -- & - & $\begin{array}{c}0,84 \\
\%\end{array}$ & $\begin{array}{c}29,5 \\
4 \\
\min \end{array}$ & $\begin{array}{l}\text { Günthner } \\
\text { et al. } \\
(2009)\end{array}$ & Praxis & $\begin{array}{c}29,54 \mathrm{~min}=100 \\
\%=1794 \mathrm{sec}\end{array}$ & genannt \\
\hline Etiketten & & & $\begin{array}{c}0,37 \\
\%\end{array}$ & & $\begin{array}{l}\text { Günthner } \\
\text { et al. } \\
(2009)\end{array}$ & $\begin{array}{l}\text { Labor- } \\
\text { tests }\end{array}$ & - & \\
\hline $\begin{array}{l}\text { Pick-by- } \\
\text { Light }\end{array}$ & & & $\begin{array}{c}0,40 \\
\%\end{array}$ & & $\begin{array}{l}\text { Günthner } \\
\text { et al. } \\
(2009)\end{array}$ & $\begin{array}{l}\text { Labor- } \\
\text { tests }\end{array}$ & - & \\
\hline $\begin{array}{l}\text { MDT mit } \\
\text { Scanner }\end{array}$ & & & $\begin{array}{c}0,36 \\
\%\end{array}$ & & $\begin{array}{l}\text { Günthner } \\
\text { et al. } \\
(2009)\end{array}$ & $\begin{array}{l}\text { Labor- } \\
\text { tests }\end{array}$ & - & \\
\hline $\begin{array}{l}\text { MDT ohne } \\
\text { Scanner }\end{array}$ & & & $\begin{array}{c}0,69 \\
\%\end{array}$ & & $\begin{array}{l}\text { Günthner } \\
\text { et al. } \\
(2009)\end{array}$ & $\begin{array}{l}\text { Labor- } \\
\text { tests }\end{array}$ & - & \\
\hline $\begin{array}{l}\text { MDT und } \\
\text { Etiketten }\end{array}$ & & & $\begin{array}{c}0,94 \\
\%\end{array}$ & & $\begin{array}{l}\text { Günthner } \\
\text { et al. } \\
(2009)\end{array}$ & $\begin{array}{l}\text { Labor- } \\
\text { tests }\end{array}$ & - & \\
\hline Beleg & & & $\begin{array}{c}0,35 \\
\%\end{array}$ & & $\begin{array}{l}\text { Günthner } \\
\text { et al. } \\
(2009)\end{array}$ & $\begin{array}{l}\text { Labor- } \\
\text { tests }\end{array}$ & - & \\
\hline
\end{tabular}


Anhang B - Anbieterrecherche: Referenzwerte aus der Praxis

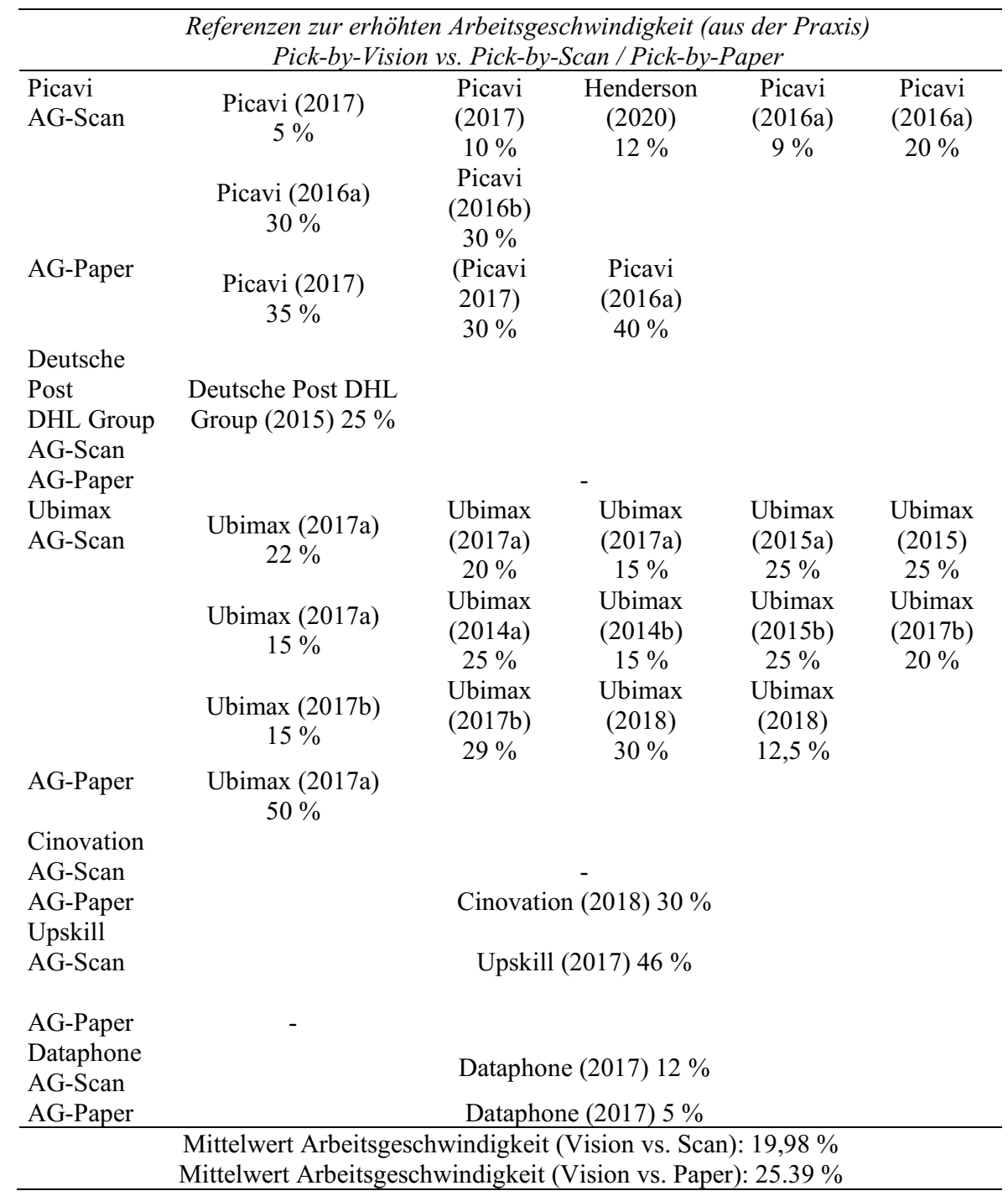

